\title{
ELLIPTIC PROBLEMS INVOLVING AN INDEFINITE WEIGHT
}

\author{
M. FAIERMAN
}

\begin{abstract}
We consider a selfadjoint elliptic eigenvalue problem, which is derived formally from a variational problem, of the form $L u=\lambda \omega(x) u$ in $\Omega$, $B_{j} u=0$ on $\Gamma, j=1, \ldots, m$, where $L$ is a linear elliptic operator of order $2 m$ defined in a bounded open set $\Omega \subset \mathbf{R}^{n} \quad(n \geq 2)$ with boundary $\Gamma$, the $B_{j}$ are linear differential operators defined on $\Gamma$, and $\omega$ is a real-valued function assuming both positive and negative values. For our problem we prove the completeness of the eigenvectors and associated vectors in two function spaces which arise naturally in such an indefinite problem. We also establish some results concerning the eigenvalues of the problem which complement the known results and investigate the structure of the principal subspaces.
\end{abstract}

\section{INTRODUCTION}

Recently there has been an increase of interest in linear elliptic boundary value problems involving an indefinite weight function due to the importance of such problems in many areas of engineering, physics, and applied mathematics. We refer to $[19,20]$ for a thorough survey of this area of investigation. In particular, if we henceforth fix our attention upon the case of selfadjoint elliptic operators, then one finds that much of the literature has been devoted to the study of the asymptotic distribution of the eigenvalues and to the derivation of their lower bounds for the case of operators having positive lower bounds. However there are other topics in the spectral theory of indefinite selfadjoint elliptic problems which still warrant investigation. For instance, if the requirement that the elliptic operator in question has positive lower bound is removed, then, as is known, there may appear nonreal or non-semi-simple eigenvalues, and there still remain questions concerning such eigenvalues which have to be answered. Moreover, the question of the completeness of the eigenvectors in certain function spaces arising naturally in indefinite problems have yet to be thoroughly dealt with. Accordingly, in this work we shall fix our attention upon some aspects of the spectral theory of indefinite selfadjoint elliptic problems of the kind just cited and, as suggested above, it will only be supposed that

Received by the editors November 14, 1987 and, in revised form, September 22, 1988.

1980 Mathematics Subject Classification (1985 Revision). Primary 35P05, 35P10, 35J25, 35J40; Secondary 46D05.

Key words and phrases. Elliptic boundary value problems, indefinite weight function, eigenvalues, eigenvectors, associated vectors, completeness, Pontrjagin space. 
the elliptic operator in question is bounded from below. In particular, we shall prove that the eigenvectors and associated vectors are complete in two function spaces which arise naturally in such problems. We shall also establish some results concerning the eigenvalues which complement the known results and investigate the structure of the principal subspaces.

We shall be concerned in this paper with a selfadjoint elliptic boundary value problem involving an indefinite weight function which is derived formally from a variational problem; and in order to present our problem we introduce the following notation. We let $x=\left(x_{1}, \ldots, x_{n}\right)$ be a generic point in $\mathbf{R}^{n}, n \geq 2$, and use the notation: $D_{k}=\partial / \partial x_{k}, D=\left(D_{1}, \ldots, D_{n}\right), D^{\alpha}=D_{1}^{\alpha_{1}} \cdots D_{n}^{\alpha_{n}}$, where $\alpha$ stands for a multi-index, $\alpha=\left(\alpha_{1}, \ldots, \alpha_{n}\right)$ whose length $\sum_{j=1}^{n} \alpha_{j}$ is denoted by $|\alpha|$. We let $\Omega$ denote a nonempty bounded open set in $\mathbf{R}^{n}$ of class $C^{m}, m \geq 1$, and let $\Gamma$ denote the boundary of $\Omega$. We also put $\mathfrak{H}=L^{2}(\Omega)$, let ( , ) and \|\| denote the inner product and norm, respectively, in $\mathfrak{H}$, let $H^{m}(\Omega)$ denote the usual Sobolev space of order $m$ related to $\mathfrak{H}$, and let $(,)_{m, \Omega}$ and \|\|$_{m, \Omega}$ denote the inner product and norm, respectively, in $H^{m}(\Omega)$. Then on $\Gamma$ we introduce the system of boundary operators (possibly void), $B_{j}(x, D)=\sum_{|\alpha| \leq m_{j}} b_{\alpha}^{j}(x) D^{\alpha}$ for $j=1, \ldots, p \leq m$, where $0 \leq m_{j} \leq$ $m-1$ and the coefficients $b_{\alpha}^{j}(x)$ are of class $C^{m-m_{j}}(\Gamma)$. We denote this system by $\left\{B_{j}\right\}_{1}^{p}, 0 \leq p \leq m$, where $p=0$ means that the system is void, and suppose henceforth that if $p>0$, then the system is normal, i.e., (i) the orders $m_{j}$ of the operators are all distinct, and (ii) $\Gamma$ is noncharacteristic to $B_{j}, j=1, \ldots, p$, at each point. Let $C^{m}\left(\bar{\Omega},\left\{B_{j}\right\}_{1}^{p}\right)$ denote $C^{m}(\bar{\Omega})$, if $p=0$ and the class of functions $u$ in $C^{m}(\bar{\Omega})$ which satisfy the boundary conditions $B_{j} u=0$ on $\Gamma$ for $j=1, \ldots, p$ if $p>0$. Let $V$ denote the closure of $C^{m}\left(\bar{\Omega},\left\{B_{j}\right\}_{1}^{p}\right)$ in $H^{m}(\Omega)$. Then in $\mathfrak{H}$ we introduce the symmetric sesquilinear form

$$
a(u, v)=\int_{\Omega} \sum_{|\alpha|,|\beta| \leq m} a_{\alpha \beta}(x) D^{\beta} u \overline{D^{\alpha} v} d x
$$

with domain $V$, where the $a_{\alpha \beta}$ are complex-valued functions satisfying $\bar{a}_{\alpha \beta}=$ $a_{\beta \alpha}, a_{\alpha \beta} \in C(\bar{\Omega})$ if $|\alpha|=|\beta|=m, a_{\alpha \beta} \in L^{\infty}(\Omega)$ otherwise, and it is also supposed that

(i) $\sum_{|\alpha|=|\beta|=m} a_{\alpha \beta}(x) \xi^{\alpha} \xi^{\beta}>0$ for all $\xi \neq 0$ in $\mathbf{R}^{n}$ and $x \in \bar{\Omega}$;

(ii) if $m>1$, then for every $x^{0} \in \Gamma$ and every $\xi \neq 0$ in $\mathbf{R}^{n}$ which is orthogonal to $\nu\left(x^{0}\right)$, where $\nu\left(x^{0}\right)$ denotes the interior unit normal to $\Gamma$ at $x^{0}$, there exists a positive constant $c=c\left(x^{0}, \xi\right)$ such that

$$
\int_{0}^{\infty} \sum_{|\alpha|=|\beta|=m} a_{\alpha \beta}\left(x^{0}\right)\left(\xi+\nu\left(x^{0}\right) \partial_{s}\right)^{\beta} f(s) \cdot \overline{\left(\xi+\nu\left(x^{0}\right) \partial_{s}\right)^{\alpha} f(s)} d s \geq c\|f\|_{H^{m}(0, \infty)}
$$

for every $f \in H^{m}(0, \infty)$ if $p=0$ and for every $f \in H^{m}(0, \infty)$ for which $\left(B_{j}^{\prime}\left(x^{0}, \xi+\nu\left(x^{0}\right) \partial_{s}\right) f\right)(0)=0$ for $j=1, \ldots, p$ if $p>0$, where $H^{m}(0, \infty)$ 
denotes the Sobolev space of order $m$ related to $L^{2}(0, \infty),\|\|_{H^{m}(0, \infty)}$ denotes the norm in $H^{m}(0, \infty), B_{j}^{\prime}(x, D)$ denotes the principal part of $B_{j}(x, D)$, and $\partial_{s}=-\mathrm{id} / d s$.

We note from [2,3] and [34, Theorem 1.1, p. 139] that $a(u, v)$ is coercive over $V$, i.e., there exist constants $c_{0}>0$ and $c_{1} \geq 0$ such that $a(u, u) \geq$ $c_{0}\|u\|_{m, \Omega}^{2}-c_{1}\|u\|^{2}$ for every $u \in V$. If $p<m$, then we shall also consider a second symmetric sesquilinear form $a^{\#}(u, v)$ with domain $V$ which we define as follows. Let $F_{j}(x, D)=\sum_{|\alpha| \leq \mu_{j}} f_{\alpha}^{j}(x) D^{\alpha}, 0 \leq \mu_{j} \leq m-1$, $j=(p+1), \ldots, m$, be a system of boundary operators which together with the $B_{j}(x, D)$ form a Dirichlet system of order $m$ on $\Gamma$, where the $f_{\alpha}^{j}$ are complex-valued functions of class $L^{\infty}(\Gamma)$ and where by a Dirichlet system of order $m$ on $\Gamma$ we mean a normal system of $m$ boundary operators on $\Gamma$ each of order $<m$. Then we put

$$
a^{\#}(u, v)=\int_{\Gamma} \sum_{j=p+1}^{m} F_{j} u \overline{F_{j} v} d \sigma \quad \text { for } u, v \in V,
$$

where $\sigma$ denotes surface measure on $\Gamma$.

Notation. In the sequel we let $B(u, v)$ denote the sesquilinear form in $\mathfrak{H}$ with domain $V$ which is defined as follows: either $B(u, v)=a(u, v)$ or $B(u, v)=$ $a(u, v)+a^{\#}(u, v)$. Clearly $B$ is symmetric, $|B(u, v)| \leq c\|u\|_{m, \Omega}\|v\|_{m, \Omega}$ for $u, v \in V$, where $c$ denotes a positive constant, $B$ is coercive over $V$, and $B$ is closed in $\mathfrak{H}[33$, p. 313]. We henceforth let $\gamma$ denote the lower bound of $B$ $[33$, p. 310] and let $A$ denote the selfadjoint operator in $\mathfrak{H}$ that is associated with $B[33$, p. 323].

Let us observe next that if we identify $\mathfrak{H}$ with its antidual, then the inclusion $V \subset \mathfrak{H}$ extends naturally to the inclusions $V \subset \mathfrak{H} \subset V^{\prime}$ and the inner product $(, \quad)$ extends to a pairing between $V$ and its antidual $V^{\prime}:(u, v), u \in V^{\prime}$, $v \in V$, where $V^{\prime}$ is the completion of $\mathfrak{H}$ with respect to the norm $|u|_{-1}=$ the supremum of $|(u, v)|$ over the set $v \in V,\|v\|_{m, \Omega} \leq 1$ (see [47, Theorem 1, p. 98]). Thus we may represent $B(u, v)$ in the form $B(u, v)=(L u, v)$, where $L \in \mathfrak{L}\left(V ; V^{\prime}\right)$, and since $V^{\prime} \subset \mathfrak{D}^{\prime}(\Omega)=$ space of distributions on $\Omega$, we see that $L u$ is the distribution $\sum_{|\alpha|,|\beta| \leq m}(-1)^{\alpha} D^{\alpha} a_{\alpha \beta}(x) D^{\beta} u$. We note also that if we let $D(A)$ denote the domain of $A$, then $D(A)=\{u \mid u \in V, L u \in \mathfrak{H}\}$ and that $A u=L u$ for $u \in D(A)$ (see [33, Theorem 2.1, p. 322]).

We are now in a position to make precise the definition of the elliptic boundary value problem under consideration here. Accordingly, let $\omega(x)$ be a realvalued, measurable function defined on $\Omega$ which takes on positive and negative values. Then we define our problem as follows: determine pairs $\{\lambda, u\}$, where $\lambda \in \mathbf{C}$ and $0 \neq u \in V$, for which

$$
B(u, v)=\lambda(\omega u, v) \text { for every } v \in V,
$$

where we suppose for the moment that $\omega u \in V^{\prime}$ for $u \in V$. On the other 
hand, it follows from [2, 3, 4] and [35, pp. 200-208] that when $\Omega$, the $a_{\alpha \beta}$, the $b_{\alpha}^{j}$, and the $f_{\alpha}^{j}$ are sufficiently smooth, and under suitable assumptions on $\omega$, the variational problem (1.2) corresponds to the selfadjoint regular elliptic boundary value problem

$$
L u=\lambda \omega(x) u \text { in } \Omega \text {, }
$$

where for $p<m$, the $B_{j}, j=(p+1), \ldots, m$, are a complementary system of boundary operators of $\left\{B_{j}\right\}_{1}^{p}$ relative to $B$ [2] (note that if $B(u, v)=$ $a(u, v)+a^{\#}(u, v)$, then we are to take $\left\{B_{j}\right\}_{1}^{p},\left\{F_{j}\right\}_{p+1}^{m}$ as the Dirichlet system of order $m$ used in determining the $\left.B_{j}, j=(p+1), \ldots, m\right)$. It is precisely the problem (1.3-4) which is the elliptic boundary value problem referred to above as the subject of investigation in this work; and since we do not assume the regularity required to arrive at this problem, it is to be interpreted in a purely formal manner. Thus in the sequel when we refer to the problem (1.3-4), it is to be understood in the sense of equation (1.2).

Let us now make precise our assumptions, referred to above, concerning $\omega(x)$. Accordingly, we note that the indefiniteness of $\omega(x)$ induces the natural decomposition $\Omega=\Omega^{+} \cup \Omega^{-} \cup \Omega^{0}$, where

$$
\begin{gathered}
\Omega^{+}=\{x \in \Omega ; \omega(x)>0\}, \quad \Omega^{-}=\{x \in \Omega ; \omega(x)<0\}, \\
\Omega^{0}=\{x \in \Omega ; \omega(x)=0\},
\end{gathered}
$$

and we henceforth assume that $\left|\Omega^{+}\right|>0,\left|\Omega^{-}\right|>0$, where || denotes $n$ dimensional Lebesgue measure. Next we let $T$ denote the operator in $\mathfrak{H}$ defined by $(T f)(x)=\omega(x) f(x)$. Then we furthermore suppose that $D(T) \supset$ $V(D(T)=$ domain of $T)$ and that with respect to the Hilbert topology of $V$ induced by the norm $\left(B(u, u)-(\gamma-1)\|u\|^{2}\right)^{1 / 2}$ (or equivalently, induced by the norm \|\|$\left._{m, \Omega}\right)$, the mapping $T: V \rightarrow \mathfrak{H}$ is continuous. We observe from [1, Theorem 5.4, p. 97] that a sufficient condition for the validity of this last supposition is that $\omega \in L^{r}(\Omega)$, where $r=n / m$ if $n / m>2, r>2$ if $n / m=2$, and $r=2$ otherwise. Lastly we note that under our assumptions $T$ is a selfadjoint operator in $\mathfrak{H}$.

We call $\lambda$ an eigenvalue of the problem (1.3-4) if $A u=\lambda T u$ for some $u \neq 0$ in $D(A) ; u$ is called an eigenvector of (1.3-4) corresponding to $\lambda$. Now suppose that $\lambda$ is an eigenvalue of (1.3-4) and let $N_{\lambda}$ denote the set of all eigenvectors of (1.3-4) corresponding to $\lambda$ together with the zero vector in $\mathfrak{H}$. Then $N_{\lambda}$ is a subspace of $\mathfrak{H}$ which is called the eigenspace of (1.34) corresponding to $\lambda$ and $\operatorname{dim} N_{\lambda}$ is called the geometric multiplicity of $\lambda$. If $0 \neq u_{0} \in N_{\lambda}$, then it may happen that there exist vectors $\left\{u_{j}\right\}_{1}^{r}$ in $D(A)$ 
satisfying $(A-\lambda T) u_{j}=T u_{j-1}$ for $j=1, \ldots, r$. Then the vectors $\left\{u_{j}\right\}_{1}^{r}$ are said to be associated with the eigenvector $u_{0}$ and the set $M_{\lambda}$ consisting of all eigenvectors of (1.3-4) corresponding to $\lambda$ together with their associated vectors and the zero vector in $\mathfrak{H}$ forms a subspace of $\mathfrak{H}$ which we call the principal subspace of (1.3-4) corresponding to $\lambda$ and $\operatorname{dim} M_{\lambda}$ is called the algebraic multiplicity of $\lambda$. If $N_{\lambda}=M_{\lambda}$, then we say that $\lambda$ is semisimple. We will see in the sequel that the eigenvalues of the problem (1.3-4) must all be semisimple if $\gamma>0$, while if $\lambda$ is a non-semi-simple eigenvalue and either $\lambda=0$ or $\lambda$ is not real, then $\operatorname{dim} M_{\lambda}$ does not exceed twice the number of nonpositive eigenvalues of $A$ counted according to multiplicity.

In $\S 2$ it will be shown that $A$ has a discrete spectrum. However, unlike the case of a definite weight, it is now not possible to assert that by means of a shift of parameter we can always arrive at the situation where $0 \in \rho(A)$, and because of this certain complications may arise. Indeed, our method requires that when $0 \in \sigma(A)$ we decompose $V$ into a direct sum of $M_{0}$ and a subspace which is either a Hilbert space or a Pontrjagin space (see $\S 2$ for terminology) with respect to the inner product $B($,$) . Hence in order to ensure that such$ a decomposition is possible, we are led to introduce some further assumptions. Accordingly, we henceforth suppose that if $\left|\Omega^{0}\right|>0,0 \in \sigma(A)$ (and this can only occur for $\gamma \leq 0)$, and the inner product $(T \cdot, \cdot)$ is degenerate on $\operatorname{ker} A$ (see $\S 2$ ), then the following conditions are satisfied: (1) $\Omega$ is a region, (2) the interiors of $\Omega^{+}$and $\Omega^{-}$are not both empty, and (3) $L$ has the unique continuation property, i.e., if $u \in D(A)$, if $L u=0$, and if $u$ vanishes almost everywhere in a nonempty open subset of $\Omega$, then $u=0$. To give sufficient conditions for the validity of this last property, let us suppose that the $a_{\alpha, \beta}$ in (1.1) also satisfy: $a_{\alpha \beta} \in C^{|\alpha|-1,1}(\Omega)$ for $|\alpha|>0$ (see [22, p. 52]). Then it follows from the definition of $L u$ above that $L$ is actually a differential operator, and moreover, if $u \in D(A)$ and $L u=0$, then we know from [5, Lemma 9.5 , p. 120] that $u \in H_{\mathrm{loc}}^{2 m}(\Omega)$. Thus under this added assumption concerning the $a_{\alpha \beta}$ we conclude from [30, Theorem 2.4] that $L$ has the unique continuation property if $m=1$, while the same is true for $m>1$ (see [29, Remark 3, p. 203 and Theorem 8.9.1, p. 224]) if we suppose furthermore that $L$ has simple characteristics. Note that for the special case where $m=p=1$ (see the remarks preceding $(1.1)$ ) and $\gamma=0, L$ has the unique continuation property under the only added assumption that the $a_{\alpha \beta}$ in (1.1) are real-valued (see [45, Appendix I], [22, Theorem 8.20, p. 199]).

Let us briefly describe the methods used in establishing our main results. If $0 \in \sigma(A)$, then we decompose $V$ into the direct sum of $M_{0}$ and a subspace $V_{0}$, while in the contrary case, we put $V=V_{0}$. Then $V_{0}$, when equipped with the inner product $B($,$) , becomes either a Hilbert space or a Pontrjagin space,$ where this latter case can only occur for $\gamma<0$. In the inner product space $V_{0}$ we introduce the compact selfadjoint operator $K$ whose characteristic values are precisely the nonzero eigenvalues of the problem (1.3-4); and by an analysis 
of the spectral properties of $K$ we are able to arrive at our main results. In all but two exceptional cases we are able to establish our results under a minimum of hypotheses and by means of techniques associated with the space concerned. The first exceptional case occurs when $\left|\Omega^{0}\right|>0,0 \in \sigma(A)$, and the inner product $(T \cdot, \cdot)$ is degenerate on $\operatorname{ker} A$ (see Theorems 2.4-5). For this case we have had to impose special restrictions on $\Omega, \omega$, and require that $L$ have the unique continuation property in order to arrive at the desired decomposition of $V$. The second exceptional case occurs when $\left|\Omega^{0}\right|>0, \gamma<0, V_{0}$ is a Pontrjagin space, and the inner product $B($,$) is degenerate on \operatorname{ker} T \cap V$ (see Theorem 4.9). For this case we can no longer arrive at a suitable decomposition of $V_{0}$ (as was achieved in Theorem 4.8) which would enable us to establish all the results which we desire. Hence for this reason we have had to abandon the theory of Pontrjagin spaces and achieve our objectives instead by appealing to the theory of compact symmetrisable transformations in Hilbert space and this has forced us to impose rather stringent assumptions on $\omega$ and on the coefficients of $L$.

Boundary value problems of the form (1.3-4), with $\omega$ changing signs in $\Omega$, have been the subject of much investigation. Fixing our attention firstly upon the situation where $L$ is an ordinary differential operator, i.e., $n=1$, we might mention the early works of Hilbert [27], who for the case $m=1$, $\gamma>0$, proved the existence of infinitely many positive and negative eigenvalues and considered the corresponding eigenfunction expansion, and of Haupt [23, 24], Hilb [26], and Richardson [41, 42, 43], who for the case $m=1$ and $\gamma$ arbitrary proved the existence of infinitely many positive and negative eigenvalues, with the last author also alluding to the possible existence of nonreal eigenvalues. For more recent works concerning the case $m=1, \gamma$ arbitrary we refer to those of Jörgens [32], who makes some conjectures about the asymptotic behaviour of the spectral distribution functions, and of Mingarelli [37, 38], who establishes bounds for the number of nonreal and non-semi-simple eigenvalues of the problem in terms of the number of negative eigenvalues of the associated elliptic operator. We might also mention at this point the lesser known work of Gerlach [21] dealing with the existence of nonreal eigenvalues of the problem and the establishment of a bound for their number. Lastly, for a comprehensive survey of the 1-dimensional problem we refer to [39]. Turning next to partial differential operators, we might mention the early works of Hilbert cited above and of Holmgren [28], who considered the Dirichlet problem for $L=-\Delta$ and proved the existence of infinitely many positive and negative eigenvalues. The asymptotic distribution of the eigenvalues of the problem considered by Holmgren (and also for Neumann boundary conditions) has been established by Pleijel [40]. For recent works concerning the asymptotic distribution of eigenvalues, we refer to Fleckinger and Lapidus $[19,20]$, who deal with the problem (1.3-4) (with Dirichlet and Neumann boundary conditions), and to Birman and Solomjak [8, 9, 10,11] and Rozenbljum [44] who deal with 
a more general problem (i.e., $\omega$ replaced by a differential operator); and for a variational characterization of the eigenvalues of this latter problem we refer to Weinberger [46, Chapter 3]. Finally, in the discussion below, some further relevant references will be given.

Let us now compare our results with those found in the literature and in this way show how they complement the known ones. Accordingly, it is our opinion that our most novel results are those concerning the completeness of the eigenvectors and associated vectors of the problem (1.3-4) in the two function spaces under consideration here (see Theorems 3.2, 4.8-9). Note that we have established completeness for a problem involving an indefinite weight and an elliptic operator which is not necessarily positive. We remark that affirmative answers to the question of completeness in the function space $L^{2}\left(\Omega^{+} \cup \Omega^{-}\right)$ have been given in [25 and 27] for certain problems for which $m=1$ and $\gamma>$ 0 , and where in [27] it is also supposed that $\left|\Omega^{0}\right|=0$. Concerning the function space $L^{2}\left(\Omega^{+} \cup \Omega^{-} ;|\omega(x)| d x\right)$, it has been shown in [6 and 16] that when $m=1, \gamma \geq 0$, and under certain other special conditions, the eigenvectors and associated vectors actually form an unconditional basis. We have, however, not dealt with this basis problem here as it would have diverted us from the main aim of the paper. Fixing our attention next upon the nonreal and non-semisimple eigenvalues of the problem (1.3-4) (see Theorems 2.4-5, 3.1, 4.2-6), we have already mentioned that the existence of such eigenvalues is known when $L$ is an ordinary differential operator and that for this case, and under certain conditions, estimates as to their number have been established. For the case of partial differential operators, and only for certain boundary value problems, some relevant results are given in [17, 18], but they are of a somewhat limited nature and not much detail is given. We have, on the other hand, established very general results concerning such eigenvalues under less restrictive conditions than hitherto supposed (e.g., in $[37,38]$ only the case $0 \in \rho(A)$ is considered); and in particular, we have established in Theorems 3.1 and 4.2 the important result that all the eigenvalues of the problem (1.3-4) are of finite algebraic multiplicity. We remark that the proof of this last result is a matter of some difficulty, due in part to the generality of the conditions supposed here (and hence, not dealt with by other authors) and in part to the possible nonuniqueness of the Cauchy problem, and indeed, due to this latter problem, the result could only be established in general for the case $\lambda=0$ an eigenvalue by invoking the unique continuation hypothesis (we refer to the arguments of $\S 2$ leading to the decomposition $V=M_{0}+V_{0}$ ). We also remark that the assertions of Theorems 4.4-5, concerning a necessary and sufficient condition for the semisimplicity of an eigenvalue, appear to be new. Turning lastly to the assertion that there are always infinitely many positive and negative eigenvalues (see Theorem 3.1, 4.8-9), this result is known for ordinary differential operators of the second order (cf. [43]). For the problem under consideration here the result is known for $\gamma>0$ (cf. [8, 9, 46]). For the general case, i.e., no restriction on $\gamma$, the 
result can be inferred from the arguments of [10], where it is supposed that the form $B$ contains no boundary integral and $\Omega$ and all the coefficients appearing in the problem (1.3-4) are of class $C^{\infty}$ (here the method of pseudodifferential operators is used). When $L=-\Delta+q,\left|\Omega^{0}\right|=0$, and Dirichlet boundary conditions are assumed, the result for the general case has also been given in [18], although the proof is only sketched. When $L=(-\Delta)^{m}$ and Neumann boundary conditions are assumed (so that $\gamma=0$ ), the result has been given in [11], and it is obtained by factoring out a finite dimensional subspace. In view of the fact that we have had to invoke the unique continuation hypothesis in order to obtain such a factorization in general, we do not quite follow how this has been achieved in [11]. Let us remark that the proof of the assertion is by no means trivial; indeed, under the hypotheses of Theorem 4.9, even the Pontrjagin space theory did not suffice in allowing us to establish it.

Finally, $\S 2$ is devoted to introducing material which is required in the sequel and to establishing the decomposition of $V$ mentioned above when $0 \in \sigma(A)$. In $\S 3$ we deal with the case where $V_{0}$ mentioned above is a Hilbert space with respect to the inner product $B($,$) , and our main results are given in Theorems$ 3.1-2. In $\S 4$ we deal with the case where $V_{0}$ is a Pontrjagin space with respect to the inner product $B($,$) , and our main results are given in Theorems 4.2-6$ and 4.8-9.

\section{Preliminary Results}

The main results referred to in $\S 1$ will depend upon certain preliminary results which we wish now to present. However, before doing this, let us, for the readers convenience, present some basic definitions concerning inner product spaces since much of the material in this paper rests upon the theory of such spaces.

A vector space $X$ over $\mathbf{C}$ in which is defined an inner product, [ , ] is called an inner product space (here [ , ] is only assumed to be a complexvalued, symmetric, and sesquilinear). If $[x, x] \geq 0$ for every $x \in X$, then $X$ is called a positive inner product space. If $X$ is a positive inner product space such that $[x, x]=0$ implies that $x=0$, then $X$ is called a positive definite inner product space. Negative and negative definite inner product spaces are defined analogously. We call $X$ a neutral inner product space if $[x, x]=0$ for every $x \in X$ and an indefinite inner product space if for some $x, y$ in $X,[x, x]>0$ and $[y, y]<0$. Analogous definitions hold for subspaces of $X$. Two subsets $X_{1}, X_{2}$ of $X$ are said to be orthogonal if $\left[x_{1}, x_{2}\right]=0$ for every $x_{1} \in X_{1}, x_{2} \in X_{2}$, while the set $\left\{x \in X \mid\left[x, x_{1}\right]=0\right.$ for every $\left.x_{1} \in X_{1}\right\}$ is called the orthogonal companion of $X_{1}$. If $X_{1}$ is a subspace of $X$ and $X_{1}^{0}$ denotes the intersection of $X_{1}$ with its orthogonal companion, then the inner product is said to be degenerate on $X_{1}$ if $X_{1}^{0} \neq 0$. If $X$ is an indefinite inner product space, then $X$ is said to be decomposable if it can be represented as the direct sum of a positive definite, a negative definite, and a neutral subspace which are pairwise orthogonal, and every decomposition of this type is called a 
fundamental decomposition of $X$. If $X$ is an indefinite inner product space, if $X$ can be represented as the direct sum of a positive definite subspace $X^{+}$ and a negative definite subspace $X^{-}$such that $X^{+}$is orthogonal to $X^{-}$, and if $X^{+}$and $X^{-}$are Hilbert spaces with respect to the inner products [, ] and $-[$,$] , respectively, then X$ is called in general a Krein space, but for the particular case where the Hilbert space dimension of $X^{+}$or $X^{-}$is finite, it is called a Pontrjagin space. For further information regarding Pontrjagin spaces we refer to [12].

Let us now turn to the main work of this section. Accordingly, we first note from the assumptions of $\S 1$ and [1, Theorem 6.2, p. 144] that the resolvent of $A$ is compact, and hence it follows that $A$ has a discrete spectrum, that $R(A)$, the range of $A$, is closed in $\mathfrak{H}$, and that $N=\operatorname{ker} A$ is finite dimensional. Note also that when $V$, considered only as a vector space, is equipped with the inner product $B($,$) , then it becomes a positive definite or a positive inner product$ space according to whether $\gamma>0$ or $\gamma \geq 0$, while we assert that if $\gamma<0$, then $V$ becomes an indefinite inner product space. This last assertion follows from an argument similar to that in [5, Lemma 11.6, p. 158] if we bear in mind that $B$ is coercive over $V$ and $V$ is dense in $\mathfrak{H}$.

Henceforth we let $l=\operatorname{dim} N$. Note that if $\gamma=0$ or if $\gamma<0$ and $0 \in \sigma(A)$, then $N=N_{0}$ (see $\S 1$ ), and hence $l$ is precisely the geometric multiplicity of the eigenvalue $\lambda=0$ of (1.3-4); in all other cases we have $l=0$. If $\gamma<0$, then we also let $l^{-}$denote the number of negative eigenvalues of $A$ counted according to multiplicity. Then supposing that $\gamma<0$ and considering $V$ as an indefinite inner product space with respect to the inner product $B($,$) , we$ have

Theorem 2.1. If $\mathfrak{L}$ is a negative definite subspace of $V$, then $\operatorname{dim} \mathfrak{L} \leq l^{-}$, while if $\mathfrak{L}$ is a negative subspace of $V$, then $\operatorname{dim} \mathfrak{L} \leq l+l^{-}$.

Proof. Let $\tau$ denote the topology on $V$ induced by the norm \|\|$_{m, \Omega}$ and let

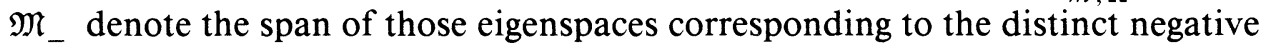
eigenvalues of $A$. Then it follows from the spectral theorem and the fact that $D(A)$ is a core of $B[33$, p. 322] that $V$ admits a fundamental decomposition having neutral component $N$ (which does not appear if $N=0$ ), negative definite component $\mathfrak{M}_{-}$, and positive definite, $\tau$-closed component $\mathfrak{M}_{+}$. If $\mathfrak{L}$ is a negative definite subspace of $V$, if $P$ denotes the projection mapping $V$ onto $\mathfrak{M}_{-}$along $N+\mathfrak{M}_{+}$, and if $P x=0$ for $x \in \mathfrak{L}$, then it follows from an argument similar to that used in the proof of Theorem 10.1 of $[12$, p. 50] that $x=0$. Hence we conclude that $\operatorname{dim} \mathfrak{L} \leq l^{-}$. Similarly we can show that if $\mathfrak{L}$ is a negative subspace of $V$, then $\operatorname{dim} \mathfrak{L} \leq l+l^{-}$.

Again considering $V$ only as a vector space, let us introduce into $V$ the inner product $(,)_{T}=(T \cdot, \cdot)$.

Theorem 2.2. Suppose that either $\gamma=0$ or $\gamma<0$ and $0 \in \sigma(A)$. Then there is a basis $\left\{v_{j}\right\}_{1}^{l}$ of $N$ such that $\left(v_{j}, v_{k}\right)_{T}=0$ for $1 \leq j, k \leq l, j \neq k$, 
$\left(v_{j}, v_{j}\right)_{T}= \pm 1$ for $j=1, \ldots, l$ if the inner product $(,)_{T}$ is not degenerate on $N$, while if $(,)_{T}$ is degenerate on $N$, then there exists the integer $i$, $1 \leq i \leq l$, such that $\left(v_{j}, v_{j}\right)_{T}=0$ for $j=1, \ldots, i$, and $\left(v_{j}, v_{j}\right)_{T}= \pm 1$ if $i<l$ and $i<j \leq l$.

Proof. If $P$ denotes the orthoprojector mapping $\mathfrak{H}$ onto $N$, then the assertions of the theorem follow immediately from the facts that $P T$ is a symmetric operator on $N$ and $\operatorname{ker} P T=0$ if and only if the inner product $(,)_{T}$ is not degenerate on $N$.

Notation. We write $\dot{+}$ for the direct sum of subspaces of $\mathfrak{H}$, while if $X$ is a subspace of $\mathfrak{H}$, then we let $X^{\perp}$ denote its orthogonal complement in $\mathfrak{H}$ with respect to the inner product $($,$) .$

Theorem 2.3. $\lambda=0$ is an eigenvalue of the problem (1.3-4) if and only if $\gamma=0$ or $\gamma<0$ and $0 \in \sigma(A)$. If $\lambda=0$ is an eigenvalue of (1.3-4), then $N_{0}=N$, and if we suppose moreover that the inner product $(,)_{T}$ is not degenerate on $N$, then we also have $N_{0}=M_{0}$ and $\mathfrak{H}=M_{0} \dot{+}\left(T M_{0}\right)^{\perp}$.

Proof. The first two assertions are clear. Hence we suppose from now on that the inner product $(,)_{T}$ is not degenerate on $N$ and let us firstly show that $N_{0}=M_{0}$. Indeed if this is not the case, then there is a $v \neq 0$ in $N$ and a $u \in D(A)$ such that $A u=T v$. Then $\left(v, v_{j}\right)_{T}=0$ for $j=1, \ldots, l$, and in light of Theorem 2.2, we arrive at the contradiction that $(,)_{T}$ is degenerate on $N$. The final assertion concerning the decomposition of $\mathfrak{H}$ follows from the fact that $\operatorname{det}\left(\left(v_{j}, v_{k}\right)_{T}\right)_{j, k=1}^{l}= \pm 1$, which also shows that the vectors $\left\{T v_{j}\right\}_{1}^{l}$ are linearly independent in $\mathfrak{H}$.

Theorem 2.4. Suppose that $\gamma=0$ and the inner product $(,)_{T}$ is degenerate on $N$. Then there exist the vectors $v_{j k} \in D(A), j=1, \ldots, l, k=0, \ldots, p_{j}$, where $p_{j}=1$ for $j=1, \ldots, i, p_{j}=0$ for $j>i$ if $i<l$, such that $\left\{v_{j 0}\right\}_{1}^{l}$ is a basis of $N$ which satisfies the assertions of Theorem 2.2 when $v_{j}$ there is replaced by $v_{j 0}, A v_{j 1}=T v_{j 0}$ for $j=1, \ldots, i,\left(v_{j k}, v_{r s}\right)_{T}=0$ if $j \neq r$, and $\left(v_{j k}, v_{j s}\right)_{T}= \pm 1$ or 0 according to whether $k+s=p_{j}$ or $k+s \neq p_{j}$. Moreover, the vectors $v_{j k}$ (resp. T $v_{j k}$ ) form a linearly independent set in $\mathfrak{H}$, $M_{0}=N \dot{+} \operatorname{span}\left\{v_{j 1}\right\}_{1}^{i}$, and $\mathfrak{H}=M_{0} \dot{+}\left(T M_{0}\right)^{\perp}$.

We note that in the above theorem $\left(v_{j k}, v_{j s}\right)_{T}=B\left(v_{j 1}, v_{j 1}\right)=1$ if $1 \leq j \leq i$ and $k+s=p_{j}$. We shall however not prove the theorem as the proof is similar to that of Theorem 2.5 below (it is easier since here $B \geq 0$ in $V$ ). Next letting $[x]$ denote the integer part of $x$, we have

Theorem 2.5. Suppose that $\gamma<0,0 \in \sigma(A)$, and the inner product $(,)_{T}$ is degenerate on $N$. Then there exist the vectors $v_{j k} \in D(A), j=1, \ldots, l$, $k=0, \ldots, p_{j}$, where $p_{1} \geq p_{2} \geq \cdots \geq p_{i} \geq 1, p_{j}=0$ for $j>i$ if $i<l$, and $\sum_{j=1}^{l}\left[p_{j} / 2\right] \leq l^{-}$, such that $\left\{v_{j 0}\right\}_{1}^{l}$ is a basis of $N$ which satisfies the assertions 
of Theorem 2.2 when $v_{j}$ there is replaced by $v_{j 0}$,

$$
\begin{aligned}
& A v_{j k}=T v_{j, k-1}\left(v_{j,-1}=0\right), \quad\left(v_{j k}, v_{r s}\right)_{T}=0 \quad \text { if } j \neq r, \text { and } \\
& \left(v_{j k}, v_{j s}\right)_{T}= \pm 1 \text { or } 0 \text { according to whether } k+s=p_{j} \text { or } k+s \neq p_{j} .
\end{aligned}
$$

Moreover, the vectors $v_{j k}\left(\right.$ resp. $\left.T v_{j k}\right)$ form a linearly independent set in $\mathfrak{H}$, and if $I$ denotes the set of tuples $(j, k), j=1, \ldots, i, k=1, \ldots, p_{j}$, then $M_{0}=N \dot{+} \operatorname{span}\left\{v_{j k}\right\}_{(j, k) \in I}$ and $\mathfrak{H}=M_{0} \dot{+}\left(T M_{0}\right)^{\perp}$.

Proof. Let $\left\{v_{j}\right\}_{1}^{l}$ be a basis of $N$ satisfying the assertions of the Theorem 2.2 and for $1 \leq j \leq i$, let $e_{j}$ be a solution of $A x=T v_{j}$ such that $\left(e_{j}, v_{k}\right)_{T}=0$ for $i<k \leq l$ if $i<l$. Let $C$ denote the matrix $\left(\left(e_{j}, v_{k}\right)_{T}\right)_{j, k=1}^{i}$. Since $C$ is Hermitian, it follows that there exist the vectors $v_{j k}^{1}, j=1, \ldots, i, k=0$, 1 , such that $\operatorname{span}\left\{v_{j 0}^{1}\right\}_{1}^{i}=\operatorname{span}\left\{v_{j}\right\}_{1}^{i}, \operatorname{span}\left\{v_{j 1}^{1}\right\}_{1}^{i}=\operatorname{span}\left\{e_{j}\right\}_{1}^{i}, A v_{j 1}^{1}=T v_{j 0}^{1}$, $\left(v_{j 1}^{1}, v_{k 0}^{1}\right)_{T}=0$ if $k \neq j$, and $\rho_{j}^{1}=\left(v_{j 1}^{1}, v_{j 0}^{1}\right)_{T}= \pm 1$ for $j=1, \ldots, i$ if $\operatorname{det} C \neq 0$, while if $\operatorname{det} C=0$, then there exists the integer $i_{1}, 1 \leq i_{1} \leq i$, such that $\rho_{j}^{1}=0$ for $j=1, \ldots, i_{1}, \rho_{j}^{1}= \pm 1$ if $i_{1}<i$ and $j>i_{1}$. If $\operatorname{det} C \neq 0$, then let $v_{i 1}=v_{i 1}^{1}+\alpha v_{i 0}^{1}, v_{i 0}=v_{i 0}^{1}$, where $\alpha$ is chosen so that $\left(v_{i 1}, v_{i 1}\right)_{T}=0$, and assuming that $v_{j k}$ has been defined for $i \geq j>p \geq 1, k=0,1$, so that (2.1) is satisfied for $p<j, r \leq i$ and $p_{j}=1$, and where $v_{j k} \in \operatorname{span}\left\{v_{r s}^{1}\right\}$, $r=j, \ldots, i, s=0,1$, let $v_{p 1}=v_{p 1}^{1}+\sum_{j=p}^{i} \alpha_{p j} v_{j 0}^{1}, v_{p 0}=v_{p 0}^{1}$, where the $\alpha_{p j}$ are chosen so that $\left(v_{p 1}, v_{j 1}\right)_{T}=0$ for $j=p, \ldots, i$. Then putting $v_{j 0}=v_{j}$ for $j=(i+1), \ldots, l$ if $i<l$, we thus arrive at the vectors $v_{j k}$ of the theorem. If $\operatorname{det} C=0$ and $i_{1}<i$, then we define $v_{j k}, j=\left(i_{1}+1\right), \ldots, i, k=0,1$, precisely as above.

Suppose that $\operatorname{det} C=0$. Then we continue with the above arguments to arrive at the following situation. There exist the integers $\{s(j)\}_{1}^{q},\left\{i_{s(j)}\right\}_{1}^{q}$, where $1 \leq q \leq i, 0 \leq s(1)<s(2)<\cdots<s(q)$, and $1 \leq i_{s(q)}<i_{s(q-1)}<$ $\cdots<i_{s(1)}=i$, vectors $v_{j k}^{p}, j=1, \ldots, i_{s(q)}, k=0, \ldots, p=s(q)+1$, contained in $D(A)$, and if $q>1$, vectors $v_{j k}, j=\left(i_{s(r+1)}+1\right), \ldots, i_{s(r)}$, $k=0, \ldots, p_{j}=s(r)+1, r=1, \ldots,(q-1)$, contained in $D(A)$ which satisfy $(2.1)$ and where $\left(v_{j k}, v_{r}\right)_{T}=0$ for $r>i$ if $i<l$, such that the $v_{j 0}^{p}$ and the $v_{j 0}$ (if $\left.q>1\right)$ are contained in $\operatorname{span}\left\{v_{j}\right\}_{1}^{i}$ and form a basis for this space, and for each $j, A v_{j k}^{p}=T v_{j, k-1}^{p}$ for $k=1, \ldots, p,\left(v_{j k}^{p}, v_{r}\right)_{T}=0$ for $r>i$ if $i<l,\left(v_{j k}^{p}, v_{r s}\right)_{T}=0$ if $q>1,\left(v_{j k}^{p}, v_{r 0}^{p}\right)_{T}=0$ if $k<p$, $\left(v_{j p}^{p}, v_{r 0}^{p}\right)_{T}=0$ if $r \neq j$, and finally either (1) $\rho_{j}^{p}=\left(v_{j p}^{p}, v_{j 0}^{p}\right)_{T}= \pm 1$ for $j=1, \ldots, i_{p-1}$ or (2) there exists the integer $i_{p}, 1 \leq i_{p} \leq i_{p-1}$, such that $\rho_{j}^{p}=0$ for $j=1, \ldots, i_{p}, \rho_{j}^{p}= \pm 1$ if $i_{p}<i_{p-1}$ and $i_{p}<j \leq i_{p-1}$. Let us fix our attention upon this last result and suppose that case (1) is valid. Then for $j=i_{p-1}$ let $v_{j k}=v_{j k}^{p}+\sum_{r<k} \alpha_{r+p-k} v_{j r}^{p}$ for $k=1, \ldots, p, v_{j 0}=v_{j 0}^{p}$, where the $\alpha_{s}$ are chosen so that $\left(v_{j k}, v_{j r}\right)_{T}= \pm 1$ or 0 according to whether 
$k+r=p$ or $k+r \neq p$ (see [36, p. 244]). Assuming next that the $v_{j k}$ have been defined for $i_{p-1} \geq j<\nu \geq 1, k=0, \ldots, p$ so that (2.1) is satisfied for $\nu<j, r \leq i_{p-1}$ and $p_{j}=p$, and where $v_{j k} \in \operatorname{span}\left\{v_{r s}^{p}\right\}, r=j, \ldots, i_{p-1}$, $s=0, \ldots, p$, let $z_{\nu k}=v_{\nu k}^{p}+\sum_{r, s} \beta_{r, s+p-k} v_{r s}$ for $k=1, \ldots, p, z_{\nu 0}=v_{\nu 0}^{p}$, where in the summation $r$ runs from $(\nu+1)$ to $i_{p-1}$ and $s$ runs from 0 to $(k-1)$, while the $\beta_{r, \mu}$ are chosen so that $\left(z_{\nu p}, v_{r s}\right)_{T}=0$ for $r=(\nu+1), \ldots$, $i_{p-1}, s=0, \ldots, p$ (note that we also have $\left(z_{\nu k}, v_{r s}\right)_{T}=0$ for $0 \leq k \leq p$ ). Then we let $v_{\nu k}=z_{\nu k}+\sum_{r<k} \delta_{r+p-k} z_{\nu r}$ for $k=1, \ldots, p, v_{\nu 0}=z_{\nu 0}$, where the $\delta_{s}$ are chosen so that $\left(v_{\nu k}, v_{\nu r}\right)_{T}= \pm 1$ or 0 according to whether $k+r=p$ or $k+r \neq p$. Hence if we put $v_{j 0}=v_{j}$ for $j=(i+1), \ldots, l$, if $i<l$, then we arrive at the vectors $v_{j k}$ of the theorem.

Suppose next that case $(2)$ is valid. Then if $i_{p}<i_{p-1}$, let us define the $v_{j k}, j=\left(i_{p}+1\right), \ldots, i_{p-1}, k=0, \ldots, p_{j}=p$ precisely as above, and for $1 \leq j \leq i_{p}$, let us define the vector $e_{j}^{p}$ as follows. If $i_{p}<i_{p-1}$ or if $i_{p}=i_{p-1}$ and $q>1$, then let $e_{j}^{p}$ be a solution of $A x=T\left(v_{j p}^{p}+\sum_{r, s} \alpha_{j r s} v_{r s}\right)$ such that

$$
\left(e_{j}^{p}, v_{k}\right)_{T}=0 \text { if } i<l \text { and } i<k \leq l \text {, }
$$

where in the summation $r$ runs from $i_{p}+1$ to $i$ and $s$ runs from 0 to $p_{r}-1$ and the $\alpha_{j r s}$ are chosen so that $\left(e_{j}^{p}, v_{r s}\right)_{T}=0$ for $\left(i_{p}+1\right) \leq r \leq i, 0 \leq s \leq p_{r}$. If $i_{p}=i_{p-1}$ and $q=1$, then let $e_{j}^{p}$ be a solution of $A x=T v_{j p}^{p}$ satisfying (2.2). Let $C_{p}$ denote the matrix $\left(\left(e_{j}^{p}, v_{k 0}^{p}\right)_{T}\right)_{j, k=1}^{i_{p}}$. Then since $C_{p}$ is Hermitian, we see that there exist the vectors $v_{j k}^{p+1}, j=1, \ldots, i_{p}, k=0, \ldots,(p+1)$, such that $\operatorname{span}\left\{v_{j 0}^{p+1}\right\}_{1}^{i_{p}}=\operatorname{span}\left\{v_{j 0}^{p}\right\}_{1}^{i_{p}}, \operatorname{span}\left\{v_{j, p+1}^{p+1}\right\}_{1}^{i_{p}}=\operatorname{span}\left\{e_{j}^{p^{p}}\right\}_{1}^{i_{p}}$, and for each $j$, $A v_{j k}^{p+1}=T v_{j, k-1}^{p+1}$ for $k=1, \ldots,(p+1),\left(v_{j k}^{p+1}, v_{r}\right)_{T}=0$ for $r>i$ if $i<l$, $\left(v_{j k}^{p+1}, v_{r s}\right)_{T}=0$ if $q>1$ or $i_{p}<i_{p-1},\left(v_{j k}^{p+1}, v_{r 0}^{p+1}\right)_{T}=0$ if $k<p+1$, $\left(v_{j, p+1}^{p+1} v_{r 0}^{p+1}\right)_{T}=0$ if $r \neq j$, and $\rho_{j}^{p+1}=\left(v_{j, p+1}^{p+1}, v_{j 0}^{p+1}\right)_{T}= \pm 1$ if $\operatorname{det} C_{p} \neq 0$, while if $\operatorname{det} C_{p}=0$, then there exists the integer $i_{p+1}, 1 \leq i_{p+1} \leq i_{p}$, such that $\rho_{j}^{p+1}=0$ for $1 \leq j \leq i_{p+1}, \rho_{j}^{p+1}= \pm 1$ if $i_{p+1}<i_{p}$ and $i_{p+1}<j \leq i_{p}$. If $\operatorname{det} C_{p} \neq 0$, then we can proceed as in case (1) above to arrive at the $v_{j k}$ of the theorem; otherwise we continue as before.

We assert that the above process must terminate after a finite number of steps. To see this, let us put $z_{j k}=v_{j k}^{p}, j=1, \ldots, i_{p-1}, k=0, \ldots, p_{j}=p$, $z_{j k}=v_{j k}, j=\left(i_{p-1}+1\right), \ldots, i, k=0, \ldots, p_{j}$, if $q>1$, and let us first show that the $z_{j k}$ and the $v_{r}, r=(i+1), \ldots, l$, if $i<l$, form a linearly independent set in $\mathfrak{H}$. For if this is not the case, then there is a nontrivial linear combination of these vectors, say $u_{1}$, such that $u_{1}=0$ in $\mathfrak{H}$. From the definition of the terms involved and our hypotheses concerning $\omega(x)$ it follows that there is a nontrivial linear combination of the $z_{j k}, k<p_{j}$, say $u_{2}$, which vanishes almost everywhere in a nonempty open subset $\Omega^{\#}$ of $\Omega$. If $u_{2}$ is a 
linear combination of only the $z_{j 0}$, then we arrive at a contradiction since it has been supposed that $L$ has the unique continuation property when $\left|\Omega^{0}\right|>0$. Otherwise we have $B\left(u_{2}, \varphi\right)=0$, and hence $\left(A u_{2}, \varphi\right)=0$, for $\varphi \in C_{0}^{\infty}\left(\Omega^{\#}\right)$, and thus it follows that there is a nontrivial linear combination of the $z_{j k}$, $k<p_{j}-1$, say $u_{3}$, which vanishes almost everywhere in $\Omega^{\#}$. By continuing this argument if necessary, we finally arrive at the contradiction that there is a nontrivial linear combination of the $z_{j 0}$ which vanishes almost everywhere in $\Omega^{\#}$. This establishes the independence in $\mathfrak{H}$ of the vectors concerned and similarly we can show that the vectors $T z_{j k}$ and the vectors $T v_{r}$, if $i<l$ and $r>i$, form a linearly independent set in $\mathfrak{H}$. On the other hand it is easy to see that the $z_{j k}, j=1, \ldots, i, k=0, \ldots,\left[p_{j} / 2\right]$ and the vectors $v_{r}$, $r=(i+1), \ldots, l$, if $i<l$, span a neutral subspace of $V$ with respect to the inner product $B($,$) . Hence we conclude from Theorem 2.1$ that we must have $\sum_{j=1}^{i}\left[p_{j} / 2\right] \leq l^{-}$, and the assertion follows.

In light of the above results we may now suppose that we have arrived at the stage where only case (1) above can hold, and thus we have established the existence of the vectors $v_{j k}$ satisfying all the assertions of the theorem except the final two. To deal with these last assertions, let $M=N+\operatorname{span}\left\{v_{j k}\right\}_{(j, k) \in I}$, where $I$ is defined in the theorem. Since it is easy to see that $\operatorname{det}\left(\left(v_{j k}, v_{r s}\right)_{T}\right) \neq$ 0 , it follows immediately that $\mathfrak{H}=M \dot{+}(T M)^{\perp}$. Hence it remains only to prove that $M=M_{0}$. Accordingly, suppose that $0 \neq u_{0} \subset N,\left\{u_{j}\right\}_{1}^{r}$ is a system of vectors associated with $u_{0}$, and there exists a $k, 1 \leq k \leq r$, such that $u_{k} \notin M, u_{j} \in M$ for $j<k$. Then $u_{k}=z_{k}+y_{k}$, where $z_{k} \in M$ and $0 \neq y_{k} \in(T M)^{\perp}$. Since $A z_{k}=T z_{k-1}$, where $z_{k-1} \in M$, and $A y_{k}=T y_{k-1}$, where $u_{k-1}=z_{k-1}+y_{k-1}$, we conclude that $y_{k-1}=0$, and hence we arrive at the contradiction that $y_{k}=0$. Thus $M_{0} \subset M$, and since clearly $M \subset M_{0}$, the proof of the theorem is complete.

If $\gamma=0$ or if $\gamma<0$ and $0 \in \sigma(A)$, then let $\mathfrak{H}_{0}=\left(T M_{0}\right)^{\perp}$. Then arguments similar to those used in [15, §7] show that: (1) $V=M_{0}+V_{0}$, where $V_{0}=V \cap \mathfrak{H}_{0}$ is a closed subspace of $V$ with respect to the norm \|\|$_{m, \Omega}$ and is dense in $\mathfrak{H}_{0}$ with respect to the norm \|\| ; (2) if $A_{0}=A \mid \mathfrak{H}_{0}$, then $D\left(A_{0}\right)=D(A) \cap \mathfrak{H}_{0}$, $D(A)=M_{0}+D\left(A_{0}\right)$, and $A_{0}: D\left(A_{0}\right) \rightarrow R\left(A_{0}\right)$ is densely defined in $\mathfrak{H}_{0}$ and closed, with $\operatorname{ker} A_{0}=0$; (3) $A M_{0}$ and $R\left(A_{0}\right)$ are closed, linearly independent subspaces of $\mathfrak{H}$ such that $R(A)=A M_{0}+R\left(A_{0}\right) ;(4) \mathfrak{H}=T M_{0}+R\left(A_{0}\right)$ and $R\left(A_{0}\right)=M_{0}^{\perp}$; and (5) the mapping $A_{0}^{-1}: R\left(A_{0}\right) \rightarrow \mathfrak{H}_{0}$ is compact. It follows from these results that if $T_{0}=T \mid V_{0}$, then $T_{0} V_{0} \subset R\left(A_{0}\right)$, and hence in $V_{0}$ we may now introduce the operator $K=A_{0}^{-1} T_{0}$. Finally in the sequel we let $B_{0}=B \mid V_{0}$.

If $\gamma>0$ or if $\gamma<0$ and $0 \in \rho(A)$, then let us write $\mathfrak{H}_{0}$ for $\mathfrak{H}, V_{0}$ for $V, B_{0}$ for $B$, and in $V_{0}$ let us introduce the operator $K=A^{-1} T_{0}$, where $T_{0}=T \mid V_{0}$. 
Theorem 2.6. $B_{0}(u, v)$ is a closed, densely defined, bounded from below symmetric form on $\mathfrak{H}_{0}$ which is coercive over $V_{0}$ and $\left|B_{0}(u, v)\right| \leq c\|u\|_{m, \Omega}\|v\|_{m, \Omega}$ for $u, v \in V_{0}$, where $c$ denotes a positive constant. Moreover, if $\gamma \geq 0$, then $B_{0}$ is positive definite on $V_{0}$, while if $\gamma<0$ and $0 \in \rho(A)$ or if $0 \in \sigma(A)$ and the inner product $(,)_{T}$ is not degenerate on $N$, then $B_{0}$ is indefinite on $V_{0}$. If $\gamma<0,0 \in \sigma(A)$, and the inner product $(,)_{T}$ is degenerate on $N$, then $B_{0}$ is either positive definite or indefinite on $V_{0}$. Finally, if $B_{0}$ is positive definite on $V_{0}$, the $B(u, u) \geq k\|u\|_{m, \Omega}^{2}$ for $u \in V_{0}$, where $k$ denotes a positive constant.

Proof. Let us firstly observe that if $\gamma<0,0 \in \sigma(A)$, and the inner product $(,)_{T}$ is not degenerate on $N$, then $B_{0}$ must be indefinite on $V_{0}$. This observation follows from the fact that if $v \in V$ and $v=v_{1}+v_{2}$, where $v_{1} \in M_{0}$ and $v_{2} \in V_{0}$, then $B(v, v)=B_{0}\left(v_{2}, v_{2}\right)$. The remaining assertions of the theorem follow from the assumptions made in $\S 1$ and arguments similar to those used in the proofs of [14, Theorem 3.6 and 15, Theorem 8.1].

Remark 2.1. Suppose that $\gamma=0$ or $\gamma<0$ and $0 \in \sigma(A)$ and let $P$ denote the orthoprojector mapping $\mathfrak{H}$ onto $\mathfrak{H}_{0}$. If $A_{0}^{\dagger}$ denotes the selfadjoint operator in $\mathfrak{H}_{0}$ that is associated with the form $B_{0}$, then arguments similar to those used in the proof of [15, Theorem 8.1] show that $A_{0}^{\dagger}=P A_{0}$ and $0 \in \rho\left(A_{0}^{\dagger}\right)$. If $\gamma>0$ or $\gamma<0$ and $0 \in \rho(A)$, then for consistency of notation we shall in the sequel let $A_{0}^{\dagger}=A$.

\section{The POSITIVE DEFINITE CASE}

We have seen in Theorem 2.6 that $B_{0}$ is either positive definite or indefinite on $V_{0}$. In this section it will always be supposed that the positive definitive alternative is valid, and we shall establish our main results for this case. We note from Theorem 2.6 that $B_{0}$ is always positive definite on $V_{0}$ when $\gamma \geq 0$ and possibly when $\gamma<0,0 \in \sigma(A)$, and the inner product $(,)_{T}$ is degenerate on $N$. Also when dealing with the case $\gamma=0$, we shall distinguish between the two cases: (a) the inner product $(,)_{T}$ is not degenerate on $N$ and (b) the inner product $(,)_{T}$ is degenerate on $N$.

Theorem 3.1. The eigenvalues of the problem (1.3-4) are each of finite algebraic multiplicity and form a denumerably infinite subset of $\mathbf{R}$ having no finite points of accumulation. Moreover, there are infinitely many positive (resp. negative) eigenvalues and each nonzero eigenvalue is semisimple.

Before proving the theorem, let us remark that the theorem together with Theorem 2.3 show that the eigenvalues of the problem (1.3-4) are all nonzero if $\gamma>0$ and all semisimple if $\gamma=0$ and case $(a)$ is valid. On the other hand we see from Theorems 2.2-5 that $\lambda=0$ must always be a non-semisimple eigenvalue if $\gamma=0$ and case (b) is valid or if $\gamma<0$, and in the former (resp. latter) case its algebraic multiplicity does not exceed $2 l$ (resp. $\left.2\left(l+l^{-}\right)\right)$. Next if we denote the nonzero eigenvalues of $(1.3-4)$ by $\left\{\lambda_{j}\right\}_{1}^{\infty}$, where each eigenvalue is repeated according to its multiplicity, then we shall 
show below that to the sequence $\left\{\lambda_{j}\right\}_{1}^{\infty}$ there corresponds the sequence of eigenvectors $\left\{u_{j}\right\}_{1}^{\infty}$ satisfying $\left(u_{j}, u_{k}\right)_{T}=\delta_{j k} \operatorname{sgn} \lambda_{j} \quad\left(\delta_{j k}=\right.$ Kronecker delta $)$ and also $\left(u_{j}, v\right)_{T}=0$ for $v \in M_{0}$ if $\gamma \leq 0$ (see Theorem 2.2-5). We are also going to show that the restrictions of these eigenvectors to the set $\Omega^{+} U$ $\Omega^{-}$together with the restrictions of the eigenvectors and associated vectors corresponding to $\lambda=0$, if any, form a complete system of vectors in certain Hilbert spaces associated with $\Omega^{+} \cup \Omega^{-}$. To this end we require the following

Defnition 3.1. We say that the weight function $\omega(x)$ satisfies the condition (C) if $\mid \Omega^{+} \backslash$ int $\Omega^{+}|=| \Omega^{-} \backslash$ int $\Omega^{-} \mid=0$, and $C_{0}^{\infty}\left(\right.$ int $\left.\Omega^{+}\right) \quad\left(\right.$ resp. $C_{0}^{\infty}\left(\right.$ int $\left.\left.\Omega^{-}\right)\right)$ is dense in $L^{2}$ (int $\left.\Omega^{+} ; \omega(x) d x\right)$ (resp. $L^{2}\left(\right.$ int $\left.\Omega^{-} ;-\omega(x) d x\right)$ ), where int $=$ interior.

Theorem 3.2. The eigenvectors of the problem (1.3-4) together with their associated vectors, if any, are complete in $L^{2}\left(\Omega^{+} \cup \Omega^{-}\right)$, and if $\omega(x)$ satisfies the condition $(\mathrm{C})$, then they are also complete in $L^{2}\left(\Omega^{+} \cup \Omega^{-} ;|\omega(x)| d x\right)$.

Proofs of Theorems 3.1-2. We shall only prove the theorems for the case $\gamma<0$; the proofs of the remaining cases are similar. Accordingly, it follows from Theorem 2.6 that when $V_{0}$, considered only as a vector space, is equipped with the inner product $\langle\rangle=,B_{0}($,$) , then it becomes a Hilbert space and \|\|_{0}$ and \|\|$_{m, \Omega}$ are equivalent norms on $V_{0}$, where $\|u\|_{0}=\langle u, u\rangle^{1 / 2}$. Moreover, if we henceforth suppose, unless otherwise stated, that $V_{0}$ is equipped with the inner product $\langle$,$\rangle , then it follows from the results of \S 2$ that $K$ is compact and selfadjoint in $V_{0}$. Hence let $P^{+}$and $P^{-}$denote the positive and negative spectral projections of $K$, put $\mathfrak{L}^{ \pm}=P^{ \pm} V_{0}$, and let us show that $\operatorname{dim} \mathfrak{L}^{ \pm}=\infty$. To this end, let us consider $V$ only as a vector space, equip it with the inner product $(,)_{T}$, and let $(\dot{+})$ denote the orthogonal direct sum of subspaces of $V[12$, p. 7]. Then it is clear that $\mathfrak{L}^{+}$(resp. $\mathfrak{L}^{-}$), if not 0 , is a positive definite (resp. negative definite) subspace of $V$, while it follows from Theorem 2.5 and [12, Corollary 11.8 , p. 26] that $M_{0}=\mathfrak{M}^{+}(\dot{+}) \mathfrak{M}^{-}$, where $\mathfrak{M}^{+}$is positive definite and $\mathfrak{M}^{-}$is negative definite. Hence we conclude that $V$ admits the fundamental decomposition, $V=\operatorname{ker} K(\dot{+}) \quad\left(\mathfrak{L}^{+}(\dot{+}) \mathfrak{M}^{+}\right) \quad(\dot{+}) \quad\left(\mathfrak{L}^{-}(\dot{+}) \mathfrak{M}^{-}\right)$. On the other hand arguments similar to those used in [13, Proposition 1.11] show that $V$ contains positive definite and negative definite subspaces of arbitrary large dimension, and so it follows from arguments similar to those used in the proof of $\left[12\right.$, Theorem 10.1, p. 50] that $\operatorname{dim} \mathfrak{L}^{ \pm}=\infty$.

Returning again to the Hilbert space $V_{0}$, it is a simple matter to show with the aid of Theorem 2.5 that the characteristic values of $K$ are precisely the nonzero eigenvalues of the problem (1.3-4), that for $\lambda$ a characteristic value of $K$, the eigenspace of $K$ corresponding to the eigenvalue $1 / \lambda$ is precisely $N_{\lambda}$, and that $N_{\lambda}=M_{\lambda}$. In light of the foregoing results, this completes the proof of Theorem 3.1. Henceforth we let $\left\{1 / \lambda_{r}^{+}\right\}_{1}^{\infty}$ and $\left\{u_{r}^{+}\right\}_{1}^{\infty}$ (resp. $\left\{1 / \lambda_{r}^{-}\right\}_{1}^{\infty}$ and $\left\{u_{r}^{-}\right\}_{1}^{\infty}$ ) denote the positive (resp. negative) eigenvalues of $K$ counted 
according to multiplicity and their corresponding orthonormalized eigenvectors, respectively. Thus the $\lambda_{r}^{ \pm}$are precisely the nonzero eigenvalues of (1.3-4) counted according to multiplicity with the $u_{r}^{ \pm}$being corresponding eigenvectors. Now let us turn to the proof of Theorem 3.2; and in proving this theorem we will make use of the fact that the $u_{r}^{ \pm}$form an orthonormal basis of $\mathfrak{L}^{+} \dot{+} \mathfrak{L}^{-}$, and hence a basis of $\mathfrak{L}^{+} \dot{+} \mathfrak{L}^{-}$with respect to the topology induced on this space by the norm \|\| . Accordingly, if $h \in \mathfrak{H}^{\dagger}=L^{2}\left(\Omega^{+} \cup \Omega^{-}\right)$and $h^{\sim}(x)=h(x)$ for $x \in \Omega^{+} \cup \Omega^{-}$and $h^{\sim}(x)=0$ in $\Omega \backslash\left(\Omega^{+} \cup \Omega^{-}\right)$, then we have $h^{\sim}=h_{0}+h_{1}$, where $h_{0} \in \mathfrak{H}_{0}$ and $h_{1} \in M_{0}$. Also for any $\varepsilon>0$, there is a $z \in V_{0}$ such that $\left\|z-h_{0}\right\|<\varepsilon$. Thus since $z=z_{0}+P^{+} z+P^{-} z$, where $z_{0} \in \operatorname{ker} K \subset \operatorname{ker} T$, we see that if \|\|$^{\dagger}$ denotes the norm in $\mathfrak{H}^{\dagger}$, then $\left\|h-h_{1}-P^{+} z-P^{-} z\right\|^{\dagger}=$ $\left\|h-h_{1}-z\right\|^{\dagger} \leq\left\|h^{\sim}-h_{1}-z\right\|<\varepsilon$, and the first assertion of Theorem 3.2 follows immediately.

To prove the second assertion let $f \in \mathfrak{H}^{\#}=L^{2}\left(\Omega^{+} \cup \Omega^{-} ;|\omega(x)| d x\right)$ and let $\varepsilon$ be any positive number. Then there is a $\varphi \in C_{0}^{\infty}$ (int $\Omega^{+} \cup$ int $\Omega^{-}$) such that $\|f-\varphi\|^{\#}<\varepsilon$, where \|\|$^{\#}$ denotes the norm in $\mathfrak{H}^{\#}$. Since $\varphi=v_{0}+v_{1}$, where $v_{0} \in V_{0}$ and $v_{1} \in M_{0}$, and since it is easy to see that $\left\langle v_{0}, y\right\rangle=0$ for $y \in \operatorname{ker} K$, it follows that $\varphi=v_{1}+P^{+} v_{0}+P^{-} v_{0}$. Hence $\left\|f-v_{1}-P^{+} v_{0}-P^{-} v_{0}\right\|^{\#}<\varepsilon$ and the second assertion of the theorem follows from the fact that $\|y\|^{\#} \leq c\|y\|_{0}$ for $y \in V_{0}$, where $c$ denotes a positive constant.

\section{THE INDEFINITE CASE}

In this section it will always be supposed that $\gamma<0$ and that $B_{0}$ is indefinite on $V_{0}$ if $0 \in \sigma(A)$ and the inner $(,)_{T}$ is degenerate on $N$. In view of Theorem 2.6 this is equivalent to the assumption that $B_{0}$ is indefinite on $V_{0}$, and it is our objective now to establish our main results for this case.

We have seen in $\S 2$ that $V_{0}$ is a Hilbert space with respect to the inner product $(,)_{m, \Omega}$ and, for the case under consideration here, that when $V_{0}$ is considered only as a vector space and is equipped with the inner product $\langle\rangle=,B_{0}($,$) ,$ then it becomes an indefinite inner product space. Let us now consider $V_{0}$ as such an indefinite inner product space and let $G$ denote the Gram operator of $\langle$,$\rangle with respect to (,)_{m, \Omega}$, so that $\langle u, v\rangle=(G u, v)_{m, \Omega}$ for $u, v \in V_{0}[12$, p. 89]. Let $P_{+}$and $P_{-}$denote the positive and negative spectral projections of $G$, put $\mathfrak{L}_{ \pm}=P_{ \pm} V_{0}$, and let $\kappa=\operatorname{dim} \mathfrak{L}_{-}$. Then referring to [12, p. 184] for terminology, we have

Theorem 4.1. $V_{0}$, when equipped with the inner product $\langle$,$\rangle , is a Pontriagin$ space of index $\kappa$, where $0<\kappa \leq l^{-}$.

Proof. Firstly we remark that the theorem asserts that $V_{0}$ is a Krein space with finite rank of indefiniteness $\kappa$ and it will be shown below that $\kappa$ is precisely the rank of negativity of $V_{0}[12, \mathrm{pp} .95,100,184]$. Let us now prove that $G$ is completely invertible on $V_{0}$, or equivalently, that $G$ has a 
bounded inverse. Indeed, if this is not the case, then there exists the sequence $u_{j} \in D\left(A_{0}^{\dagger}\right)$ (see Remark 2.1) with $\left\|u_{j}\right\|_{m, \Omega}=1$ such that $\left\|G u_{j}\right\|_{m, \Omega} \rightarrow 0$. Since $\left(G u_{j}, v\right)_{m, \Omega}=\left\langle u_{j}, v\right\rangle=\left(u_{j}, A_{0}^{\dagger} v\right)$ for $v \in D\left(A_{0}^{\dagger}\right)$, it follows that $u_{j}$ has a subsequence which converges to zero in $\mathfrak{H}_{0}$. Consequently, if we denote this subsequence again by $\left\{u_{j}\right\}$ and make use of the fact that $B_{0}$ is coercive over $V_{0}$, then we arrive at the contradiction that $\left\|u_{j}\right\|_{m, \Omega} \rightarrow 0$.

Thus it follows from [12, Theorem 1.3, p. 101] that $V_{0}$ is a Krein space and admits a fundamental decomposition with components $\mathfrak{L}_{ \pm}$. On the other hand we know from Theorem 2.1 that $\kappa \leq l^{-}$, while the arguments of $\S 2$ show that $\mathfrak{L}_{+}$cannot be finite dimensional. Hence, in light of the results of [12, p. 184], the theorem is proved.

Turning next to the operator $K$ introduced in $\S 2$, it is easy to see that $K$ is a selfadjoint operator in the Pontrjagin space $V_{0}[12, \mathrm{p} .133]$ which is compact with respect to the strong topology of $V_{0}[12, \mathrm{p}$. 102] (note that the Hilbert topology on $V_{0}$ induced by the norm \|\|$_{m, \Omega}$ coincides with the strong topology of $V_{0}$ ). We are now going to use these facts to derive some information concerning the eigenvalues and principal subspaces of the problem (1.3-4). Accordingly, if we consider $V$ (resp. $V_{0}$ ) only as a vector space and equip it with the inner product $(,)_{T}$, then we may argue as in the proofs of Theorem 3.1 and [15, Theorem 8.1] to show that it becomes an indefinite inner product space.

Terminology. In the sequel, when we refer to $V_{0}$ as a Pontrjagin space, then this will always be meant with respect to the inner product $\langle$,$\rangle , while if we$ refer to $V_{0}$ (resp. $V$ ) as an indefinite inner product space, then this will always be meant with respect to the inner product $(,)_{T}$.

If we now fix our attention upon the indefinite inner product space $V$ and employ the terminology of $[12$, p. 21$]$, then we have

Theorem 4.2. The eigenvalues of the problem (1.3-4) form an at most denumerably infinite subset of $\mathbf{C}$ having no finite points of accumulation such that if $\lambda$ is an eigenvalue, then $\operatorname{dim} M_{\lambda}<\infty$. Moreover, if $\lambda$ and $\mu$ are eigenvalues satisfying $\lambda \neq \bar{\mu}$, then $M_{\lambda}$ is orthogonal to $M_{\mu}$, and hence the principal subspace corresponding to a nonreal eigenvalue is neutral. Also if $\lambda$ is an eigenvalue, then so is $\bar{\lambda}$ and $M_{\lambda}$ and $M_{\bar{\lambda}}$ form a dual pair; thus if $\lambda$ is real, then $M_{\lambda}$ is nondegenerate. Finally, the eigenspaces corresponding to the distinct eigenvalues of the problem (1.3-4) are definite, with the possible exception of at most a finite number of them, and hence the eigenvalues of (1.3-4) are real and semisimple, with the possible exception of at most a finite number of them.

Proof. Let us turn again to the operator $K$ acting in the Pontrjagin space $V_{0}$ and henceforth agree to denote the principal subspace corresponding to the eigenvalue $\lambda$ of $K$ by $\mathfrak{G}_{\lambda}$. Then it follows that $\operatorname{dim} \mathfrak{G}_{\lambda}<\infty$ if $\lambda \neq 0$. We are now going to show that the characteristic values of $K$ are precisely the nonzero eigenvalues of the problem (1.3-4) and for each characteristic value $\lambda$ we have 
$G_{1 / \lambda}=M_{\lambda}$. Accordingly, let us firstly suppose that $\lambda \neq 0$ is an eigenvalue of the problem (1.3-4). Then it is a simple matter to show with the aid of Theorems 2.3 and 2.5 that $M_{\lambda} \subset V_{0}$. Moreover, it is also clear that $N_{\lambda}=\operatorname{ker}(I-\lambda K)$. Now suppose that $\lambda$ is not semisimple and $u \in M_{\lambda}, u \notin N_{\lambda}$. Then there exist the vectors $\left\{u_{k}\right\}_{0}^{p-1}$ in $M_{\lambda}$, where $p>1, u_{p-1}=u$, and $u_{0} \neq 0$, such that $(A-\lambda T) u_{k}=T u_{k-1}$, where $u_{-1}=0$. If we now define the vectors $\left\{z_{k}\right\}_{0}^{p-1}$ by putting $\nu=-\lambda^{-2}$ and

$$
\begin{gathered}
u_{k}=\nu^{k} z_{k} \quad \text { for } k=0,1, \\
u_{k}=\nu^{k} z_{k}+\sum_{r=1}^{k-1} \alpha_{k r} z_{r} \quad \text { for } 2 \leq k \leq p-1 \text { if } p>2,
\end{gathered}
$$

where $\alpha_{k r}=\nu^{r}(-1 / \lambda)^{k-r}{ }_{(k-1)} C_{(r-1)}$ and ${ }_{p} C_{q}$ is the binomial coefficient, then $\left(K-\lambda^{-1} I\right) z_{k}=z_{k-1}$, where $z_{-1}=0$. It follows that $\left(K-\lambda^{-1} I\right)^{p} u=0$, and hence we conclude that $M_{\lambda} \subset \mathfrak{G}_{1 / \lambda}$. Conversely, if $\lambda$ is a characteristic value of $K$ such that $1 / \lambda$ is not semisimple and if $\left(K-\lambda^{-1} I\right)^{p} z=0,\left(K-\lambda^{-1} I\right)^{p-1} z \neq$ 0 , where $p>1$, then let us put $\left(K-\lambda^{-1} I\right) z_{k}=z_{k-1}$ for $k=0, \ldots,(p-1)$, where $z_{p-1}=z, z_{-1}=0$, and define the vectors $\left\{u_{k}\right\}_{0}^{p-1}$ according to (4.1). Then it follows that $(A-\lambda T) u_{k}=T u_{k-1}$ for $k=0, \ldots,(p-1)$, where $u_{-1}=0$, and hence $z \in M_{\lambda}$. Thus $\mathfrak{G}_{1 / \lambda} \subset M_{\lambda}$ and so $\mathfrak{G}_{1 / \lambda}=M_{\lambda}$. In light of the properties of $K$ cited above, the results of $\S 2$ and [12, pp. 35 and 134], and the equality

$$
(u, v)_{T}=\langle K u, v\rangle \quad \text { for } u, v \in V_{0},
$$

all but the final assertions of the theorem now follow. On the other hand, the final assertions of the theorem readily follow from arguments similar to those used in the proof of [15, Theorem 8.5], and from [12, Lemma 3.8, p. 35].

From the above proof and [12, Theorems 4.6 and 4.8, p. 191] we now have

Theorem 4.3. The number of nonzero, non-semi-simple eigenvalues of the problem (1.3-4) does not exceed $\kappa$. Moreover, the number of nonreal eigenvalues, where each such eigenvalue is repeated according to its algebraic multiplicity, does not exceed $2 \kappa$.

In the following two theorems we again fix our attention upon the indefinite inner product space $V$.

Theorem 4.4. Suppose that $\lambda$ is a nonreal eigenvalue of the problem (1.3-4). Then $\lambda$ (resp. $\bar{\lambda}$ ) is semisimple if and only if $N_{\lambda}$ and $N_{\bar{\lambda}}$ form a dual pair. Moreover, whether $\lambda$ (resp. $\bar{\lambda}$ ) is semisimple or not, $M_{\lambda}+M_{\bar{\lambda}}$ may be decomposed into the orthogonal direct sum of subspaces $\left\{\mathfrak{L}_{j}\right\}_{1}^{q}$, where each $\mathfrak{L}_{j}$ is the direct sum of two subspaces having coordinate systems $\left\{u_{j k}^{+}\right\}$and $\left\{u_{j k}^{-}\right\}, k=$ $0, \ldots,(p(j)-1)$, respectively, which satisfy: (1) $\left(A-\lambda^{ \pm} T\right) u_{j k}^{ \pm}=T u_{j, k-1}^{ \pm}$(here 
$\left.u_{j,-1}^{ \pm}=0, \lambda^{+}=\lambda, \lambda^{-}=\bar{\lambda}\right)$ and (2) $\left(u_{j k}^{+}, u_{j s}^{-}\right)_{T}=1$ or 0 according to whether $k+s=p(j)-1$, or $k+s \neq p(j)-1$, and where $p(1) \geq p(2) \geq \cdots \geq p(q) \geq 1$ and $\sum_{j=1}^{q} p(j) \leq \kappa$.

Proof. Let us now consider $V_{0}$ as a Pontrjagin space and fix our attention upon the operator $K$ acting in $V_{0}$. Then it follows from the assertions given in the proof of Theorem 4.2 and [36, pp. 242-244] that $\mathfrak{G}_{1 / \lambda}+\mathfrak{G}_{1 / \bar{\lambda}}$ may be decomposed into the orthogonal direct sum of subspaces $\left\{\mathfrak{L}_{j}\right\}_{1}^{q}$ which are invariant under $K$; moreover, each of these subspaces has a positive normal coordinate system in which the matrix of the transformation $K$ decomposes into two Jordan blocks with elementary divisors of the form $(\zeta-1 / \lambda)^{p(j)},(\zeta-1 / \bar{\lambda})^{p(j)}$. If in $\mathfrak{L}_{j}$ we denote this coordinate system by $\left\{z_{j k}^{ \pm}\right\}, k=0, \ldots,(p(j)-1)$, where $\left(K-\left(\lambda^{ \pm}\right)^{-1} I\right) z_{j k}^{ \pm}=z_{j, k-1}^{ \pm}, z_{j,-1}^{ \pm}=0, \lambda^{+}=\lambda, \lambda^{-}=\bar{\lambda}$, then it follows from the definitions of the $z_{j k}^{ \pm}$that $1 / \lambda$ (resp. $1 / \bar{\lambda}$ ) is a semisimple eigenvalue of $K$ if and only if $\operatorname{ker}(I-\lambda K)$ and $\operatorname{ker}(I-\bar{\lambda} K)$ form a dual pair. In light of (4.2) and the facts that $N_{\lambda^{ \pm}}=\operatorname{ker}\left(I-\lambda^{ \pm} K\right)$ and $M_{\lambda^{ \pm}}=\mathfrak{G}_{1 / \lambda^{ \pm}}$, the first assertion of the theorem follows immediately.

To prove the remaining assertions, let us define the vectors $\left\{w_{j, k}^{ \pm}\right\}_{0}^{p(j)-1}$ by taking $p=p(j), \nu=-\left(1 / \lambda^{ \pm}\right)^{2}, \alpha_{k r}=(-1)^{k}\left(1 / \lambda^{ \pm}\right)_{(k-1)}^{k+r} C_{(r-1)}$ and $z_{r}=z_{j r}^{ \pm}$ in (4.1) and letting $w_{j k}^{ \pm}=u_{k}$. Noting that $\left(w_{j k}^{+}, w_{j, s}^{-}\right)_{T}$ equals 0 if $k+s<$ $p(j)-1$ and equals $(1 / \lambda)\left(-1 / \lambda^{2}\right)^{p(j)-1}$ if $k+s=p(j)-1$, let us now put $u_{j k}^{-}=w_{j k}^{-}, u_{j k}^{+}=\sum_{r=0}^{k} \alpha_{p(j)-1+r-k} w_{j r}^{+}$for $k=0, \ldots,(p(j)-1)$, where $\alpha_{\nu}$ are chosen so that $\left(u_{j k}^{+}, u_{j s}^{-}\right)_{T}=1$ or 0 according to whether $k+s=p(j)-1$ or $k+s \neq p(j)-1$. Since it is a simple matter to show that the $u_{j k}^{ \pm}$satisfy the assertions made in the theorem, we may appeal to [12, Theorem 4.6, p. 191] to complete the proof of the theorem.

Arguments similar to those used in the proof of Theorem 4.4 together with [12, Theorem 4.9, p. 191] also show that

Theorem 4.5. Suppose that $\lambda \neq 0$ is a real eigenvalue of the problem (1.3-4) such that $N_{\lambda}$ is not definite with respect to the inner product $(,)_{T}$. Then $\lambda$ is semisimple if and only if the inner product $(,)_{T}$ is not degenerate on $N_{\lambda}$. Moreover, whether $\lambda$ is semisimple or not, $M_{\lambda}$ may be decomposed into the orthogonal direct sum of subspaces $\left\{\mathfrak{L}_{j}\right\}_{1}^{s}$, where each $\mathfrak{L}_{j}$ has a coordinate system $\left\{u_{j k}\right\}, k=0, \ldots,(p(j)-1)$, which satisfies: (1) $(A-\lambda T) u_{j k}=T u_{j, k-1}$ (here $\left.u_{j,-1}=0\right)$ and (2) $\left(u_{j k}, u_{j r}\right)_{T}= \pm 1$ or 0 according to whether $k+r=$ $p(j)-1$ or $k+r \neq p(j)-1$, and where $2 \kappa+1 \geq p(1) \geq p(2) \geq \cdots \geq p(s) \geq 1$.

Remark 4.1. We note from (4.2) that if $\lambda \neq 0$ is an eigenvalue of the problem (1.3-4), then $N_{\lambda}$ is definite with respect to the inner product $(,)_{T}$ if and only if it is definite with respect to the inner product $\langle$,$\rangle , and it is also$ clear (see Theorem 4.2) that if $N_{\lambda}$ is definite in either sense, then $\lambda$ must be real and semisimple. Moreover, $N_{\lambda}$ is positive definite (resp. negative definite) 
with respect to the inner product $\langle$,$\rangle if and only if \operatorname{sgn}(u, u)_{T}=\operatorname{sgn} \lambda$ (resp. $\left.\operatorname{sgn}(u, u)_{T}=-\operatorname{sgn} \lambda\right)$ for every $u \neq 0$ in $N_{\lambda}$ and we can always choose a basis $\left\{u_{j}\right\}_{1}^{r}$ of $M_{\lambda}=N_{\lambda}$ satisfying $\left(u_{j}, u_{k}\right)_{T}=\delta_{j k} \operatorname{sgn} \lambda\left(\operatorname{resp} .\left(u_{j}, u_{k}\right)_{T}=\right.$ $\left.-\delta_{j k} \operatorname{sgn} \lambda\right)$, where $\delta_{j k}=$ Kronecker delta.

Referring again to Theorem 4.5, let us firstly note from (4.2) that the $\mathfrak{L}_{j}$ are also pairwise orthogonal subspaces of the Pontrjagin space $V_{0}$. Now if $p(1)>1$, let $j^{*}$ denote the largest value of $j, 1 \leq j \leq s$, for which $p(j)>1$ and let $M_{\lambda}^{0}$ denote the span of the subspaces $\left\{\mathfrak{L}_{j}\right\}_{1}^{j^{*}}$. If $p(1)=1$, then put $M_{\lambda}^{0}=0$. If $p(s)=1$, then let $M_{\lambda}^{-}$denote the span of those $\mathfrak{L}_{j}$ for which $p(j)=1$ and $\left(u_{j 0}, u_{j 0}\right)_{T}=-\operatorname{sgn} \lambda$ (i.e., $\left\langle u_{j 0}, u_{j 0}\right\rangle<0$ ) if such $\mathfrak{L}_{j}$ exist, and put $M_{\lambda}^{-}=0$ otherwise. If $p(s)>1$, then put $M_{\lambda}^{-}=0$. Then $M_{\lambda}^{0}$ and $M_{\lambda}^{-}$ are pairwise orthogonal subspaces of the Pontrjagin space $V_{0}$ and in this latter space $M_{\lambda}^{-}$, if not 0 , is negative definite. Moreover, it readily follows from the foregoing results and from arguments similar to those of [12, Theorem 4.3, p. 190] that

Theorem 4.6. $\left[\sum\left(\operatorname{dim} M_{\lambda}^{0}+\operatorname{dim} M_{\lambda}^{-}\right)+\sum \operatorname{dim} M_{\lambda}\right] \leq 3 \kappa$, where the first summation is over all $\lambda$ satisfying the hypotheses of Theorem 4.5 and the second summation is over all nonzero eigenvalues $\lambda$ of the problem (1.3-4) for which either $\lambda$ is not real or $\lambda$ is real and $N_{\lambda}$ is negative definite with respect to the inner product $\langle$,$\rangle , and where the first or second summation is to be omitted if$ eigenvalues of the kinds asserted do not exist.

If the problem (1.3-4) has nonreal eigenvalues, then let $M^{+}$(resp. $M^{-}$) denote the span of all the $M_{\lambda}$ for which $\operatorname{Im} \lambda>0$ (resp. $\left.\operatorname{Im} \lambda<0\right)$; otherwise let $M^{+}=M^{-}=0$. If the problem $(1.3-4)$ has real nonzero eigenvalues whose corresponding eigenspaces are not definite in the indefinite inner product space $V$ (and hence in the Pontrjagin space $V_{0}$ ), then let $M^{\dagger}$ denote the span of the $M_{\lambda}$ corresponding to such eigenvalues; otherwise let $M^{\dagger}=0$. If the problem (1.3-4) has real nonzero eigenvalues whose corresponding eigenspaces are negative definite subspaces of the Pontrjagin space $V_{0}$, then let $M^{\#}$ denote the span of the $M_{\lambda}$ corresponding to such eigenvalues; otherwise let $M^{\#}=0$.

Notation. We let $M=M^{+} \dot{+} M^{-} \dot{+} M^{\dagger} \dot{+} M^{\#}$, let $\langle\dot{+}\rangle$ (resp. $(\dot{+})$ ) denote orthogonal direct sum of subspaces of the Pontrjagin space $V_{0}$ (resp. of the indefinite inner product space $V$ ), and let $V_{1}$ denote the orthogonal companion of $M$ in the Pontrjagin space $V_{0}$ (see $[12$, p. 7]).

It follows easily from the foregoing results, [12, Corollary 11.9, p. 26], and arguments similar to those used in the proof of [15, Theorem 8.6] that $V_{0}=$ $M\langle\dot{+}\rangle V_{1}$ (resp. $\left.V_{0}=M(\dot{+}) V_{1}\right)$, that $K$ is reduced by this decomposition, and that $\operatorname{ker} K \subset V_{1}$. Moreover, it is not difficult to show that $\mathfrak{H}_{0}=M \dot{+} \mathfrak{H}_{1}$, where $\mathfrak{H}_{1}=(T M)^{\perp} \cap \mathfrak{H}_{0}, V_{1}=V_{0} \cap \mathfrak{H}_{1}$, and that $V_{1}$ is a closed, nondegenerate subspace of the Pontrjagin space $V_{0}$ which is dense in $\mathfrak{H}_{1}$ with respect to the norm \|\| . Note also from Theorems $4.4-5$ and (4.2) that if $M \neq 0$ and 
$M^{\#}=0$, then $M$ is an indefinite subspace of the indefinite inner product space (resp. Pontrjagin space) $V_{0}$. From [12, Corollary 2.3, p. 186] and arguments similar to those used in the proof of Theorem 4.1 we now have

Theorem 4.7. $V_{1}$, consider as a subspace of the Pontrjagin space $V_{0}$, is also a Pontrjagin space whose index $\kappa_{1}$ satisfies $0 \leq \kappa_{1} \leq \kappa$, and $\kappa_{1}$ is precisely the rank of negativity of $V_{1}$.

We remark that, as for the case of $V_{0}$, the Hilbert topology of $V_{1}$ induced by the norm \|\|$_{m, \Omega}$ coincides with the strong topology of $V_{1}$. Note also that $K$ is a compact selfadjoint operator in the Pontrjagin space $V_{1}$ whose eigenvalues, if any, must necessarily be real and the eigenspaces of $K$ corresponding to its nonzero eigenvalues are positive definite.

We are now in a position to establish some further results concerning the spectral properties of the problem (1.3-4).

Theorem 4.8. Suppose that $\operatorname{ker} T \cap V$ is not degenerate with respect to the inner product $B($,$) . Then the problem (1.3-4) has infinitely many positive (resp.$ negative) eigenvalues. Moreover, the eigenvectors of (1.3-4) together with their associated vectors, if any, are complete in $L^{2}\left(\Omega^{+} \cup \Omega^{-}\right)$, and if $\omega(x)$ satisfies the condition (C) of $\S 3$, then they are also complete in $L^{2}\left(\Omega^{+} \cup \Omega^{-} ;|\omega(x)| d x\right)$.

Remark 4.2. Let $\mathfrak{L}=\operatorname{ker} T \cap V$. Then the hypothesis of the theorem is certainly satisfied if $\mathfrak{L}=0$, and this is always the case if $\left|\Omega^{0}\right|=0$ (see $\S 1$ ). Moreover, it is easy to see from the results of $\S 2$, Theorems $4.4-5$, and Remark 4.1 that $\mathfrak{L}=\operatorname{ker} T \cap V_{1}=\operatorname{ker} K$, and hence it follows that the hypothesis of the theorem is equivalent to the requirement that $\operatorname{ker} K$ be a nondegenerate subspace of the Pontrjagin space $V_{1}$ (it is of course a closed subspace). It follows immediately that the hypothesis of the theorem is satisfied when $\kappa_{1}=0$ since in this case $V_{1}$ is a Hilbert space with respect to the inner product $\langle$,$\rangle . Note also that it is in$ this latter formulation that we recognize our hypothesis as being the sufficient condition given in [7 and 31] for the completeness of the root vectors of $K$ in the Pontrjagin space $V_{0}$.

Proof of Theorem 4.8. We already know that $V_{1}$ is a Hilbert space with respect to the inner product $\langle$,$\rangle if \kappa_{1}=0$. Let us now show that if $\kappa_{1}>0$, then the Pontrjagin space $V_{1}$ admits a fundamental decomposition $V_{1}=\mathfrak{L}^{+}\langle\dot{+}\rangle \mathfrak{L}^{-}$with positive definite (resp. negative definite), intrinsically complete component $\mathfrak{L}^{+}$ (resp. $\mathfrak{L}^{-}$) such that $\mathfrak{L}^{-} \subset \operatorname{ker} K$ and $\mathfrak{L}^{+}$is invariant under $K$. Accordingly, suppose that $\kappa_{1}>0$ and assume firstly that $\mathfrak{L} \neq 0$. Then it follows from [12, Corollary 2.3, p. 186] that $\mathfrak{L}=\mathfrak{L}_{+}\langle\dot{+}\rangle \mathfrak{L}_{-}$, where $\mathfrak{L}_{+}$(resp. $\mathfrak{L}_{-}$), if not 0 , is positive definite (resp. negative definite), and $0 \leq \operatorname{dim} \mathfrak{L}_{-} \leq \kappa_{1}$ [12, Lemma 1.1 p. 184]. Next let $\mathfrak{L}^{\#}=\mathfrak{L}$ - if $\mathfrak{L} \neq 0$ and $\mathfrak{L}^{\#}=0$ otherwise. Then we know from [12, Theorem 2.1, p. 165 and Theorem 3.2, p. 169] that $K$, as an operator acting on the Pontrjagin space $V_{1}$, has an invariant maximal negative subspace $\mathfrak{L}^{-}$containing $\mathfrak{L}^{\#}$, while from [12, Lemma 1.2, p. 184] we also know 
that $\operatorname{dim} \mathfrak{L}^{-}=\kappa_{1}$. Since $\sigma\left(K \mid \mathfrak{L}^{-}\right)$consists precisely of the point $\lambda=0$, we conclude that the case $\mathfrak{L}^{\#}=0$ is not possible, and so $\mathfrak{L}_{-}=\mathfrak{L}^{\#} \neq 0$. Now suppose that the inner product $\langle$,$\rangle is degenerate on \mathfrak{L}^{-}$. Then there is a $u \neq 0$ in $\mathfrak{L} \cap \mathfrak{L}^{-}$such that $\langle u, v\rangle=0$ for every $v \in \mathfrak{L} \cap \mathfrak{L}^{-}$, and hence in light of the decomposition of $\mathfrak{L}$ given above we arrive at a contradiction. Thus it follows that $\lambda=0$ is a semisimple eigenvalue of $K$ and $\mathfrak{L}^{-}$is negative definite. If we now let $\mathfrak{L}^{+}$denote the orthogonal companion of $\mathfrak{L}^{-}$in the Pontrjagin space $V_{1}$, then it follows from [12, Lemma 2.1, p. 186 and Theorem 7.1, p. 112] and the fact that $K$ is selfadjoint, that $\mathfrak{L}^{+}\langle\dot{+}\rangle \mathfrak{L}^{-}$is precisely the fundamental decomposition of $V_{1}$ asserted above.

In light of the foregoing results we may now argue as we did in the proofs of Theorems 3.1-2 to complete the proof of the theorem.

In order to deal with the case where the inner product $B($,$) is degenerate$ on $\operatorname{ker} T \cap V$, we shall need a preliminary result. Accordingly, in the following lemma we again fix our attention upon the Pontrjagin space $V_{1}$, assuming now that $\kappa_{1}>0$, and we consider $K$ as an operator acting in $V_{1}$.

Lemma 4.1. Suppose that either $\mathfrak{N}_{0}=0$ or $\mathfrak{N}_{0}$ is a finite dimensional positive definite subspace of $V_{1}$ which is invariant under $K$. Let $\mathfrak{N}$ denote the orthogonal companion of $\mathfrak{N}_{0}$ in $V_{1}$. Then for any natural number $p$ there is a $u \in \mathfrak{N}$ such that $\left\langle K^{p} u, K^{p} u\right\rangle \neq 0$.

Proof. Suppose that the lemma is false. Then it follows from [12, Lemma 2.2, p. 5] that $K^{2 p} u=0$ for $u \in \mathfrak{N}$. Hence we conclude from arguments similar to those used in the proof of $[12$, Theorem 4.3, p. 190] that $\mathfrak{N}$ can be decomposed into an orthogonal direct sum of the form $\mathfrak{N}=\mathfrak{N}_{1}\langle\dot{+}\rangle \mathfrak{N}_{2}$, where $\mathfrak{N}_{1}$ is nonzero, finite dimensional, and invariant under $K$, while $\mathfrak{N}_{2}$ is closed, positive definite, and contained in $\operatorname{ker} K$. In light of the results of $\S 2$, Theorems 4.2-6, (4.2), and $[12$, Corollary 11.8, p. 26$]$ it now follows that the indefinite inner product space $V$ (we refer to the terminology preceding Theorem 4.2) admits a fundamental decomposition with finite dimensional positive definite and negative definite components. Since $V$ contains positive definite and negative definite subspaces of arbitrary large dimension, we may argue as in the proof of [12, Theorem 10.1, p. 50] to arrive at a contradiction.

Referring again to Remark 4.2 , let us now suppose that $\mathfrak{L}$ is degenerate with respect to the inner product $B(,$,$) (note that this case can only occur when$ $\left.\left|\Omega^{0}\right|>0\right)$. Then in Theorem 4.7 we must have $\kappa_{1}>0$. Moreover, it follows from [12, Theorem 3.1, p. 103] that $\mathfrak{L}$, considered as a subspace of the Pontrjagin space $V_{1}$, admits a fundamental decomposition $\mathfrak{L}=\mathfrak{L}_{0}\langle\dot{+}\rangle \mathfrak{L}_{+}\langle\dot{+}\rangle \mathfrak{L}_{-}$, where $\mathfrak{L}_{+}$(resp. $\left.\mathfrak{L}_{-}\right)$, if not 0 , is uniformly positive definite (resp. uniformly negative definite) [12, Lemma 2.1, p. 186], $\mathfrak{L}_{0}$ is neutral, $\operatorname{dim} \mathfrak{L}_{0}>0$, and $0<\operatorname{dim}\left(\mathfrak{L}_{0}\langle\dot{+}\rangle \mathfrak{L}_{-}\right) \leq \kappa_{1}$. Hence if we consider $K$ as an operator acting in the Pontrjagin space $V_{1}$, then we assert that because of these facts every maximal negative subspace of $V_{1}$ which is invariant under $K$ must be degenerate. In- 
deed, if $\mathfrak{L}^{\#}$ is such a subspace and $\mathfrak{L}^{\#}$ is not degenerate, then we may make use of the fact that $\sigma\left(K \mid \mathfrak{L}^{\#}\right)$ consists precisely of the point $\lambda=0$ to conclude that $\mathfrak{L}^{\#}$ is negative definite and $\mathfrak{L}^{\#} \subset \mathfrak{L}$. Hence by appealing to the fundamental decomposition of $\mathfrak{L}$ cited above and by arguing as in the proof of [12, Theorem 10.1, p. 50], it readily follows that $\operatorname{dim} \mathfrak{L}^{\#} \leq \operatorname{dim} \mathfrak{L}_{-}$. Since $\operatorname{dim} \mathfrak{L}^{\#}=\kappa_{1}[12$, Lemma 1.2, p. 184], we thus arrive at a contradiction.

Next let us again consider $K$ as an operator acting in the Pontrjagin space $V_{1}$. Then we know from [12, Theorem 2.1, p. 165 and Theorem 3.2, p. 169] that $K_{1}$ has an invariant maximal negative subspace $\mathfrak{L}^{-}$containing $\mathfrak{L}_{0}\langle\dot{+}\rangle \mathfrak{L}_{-}$ and an invariant maximal positive subspace $\mathfrak{L}^{+}$which are orthogonal companions of each other. Moreover, $\mathfrak{L}^{-}$is a degenerate subspace of $V_{1}, \sigma\left(K \mid \mathfrak{L}^{-}\right)$ consists precisely of the point $\lambda=0$, and $\mathfrak{L}^{-}$is precisely the principal subspace of $K \mid \mathfrak{L}^{-}$corresponding to the eigenvalue $\lambda=0$. Let $p(\lambda)$ denote the minimum polynomial of $K \mid \mathfrak{L}^{-}$. Then $p(\lambda)=\lambda^{q}$, where $1 \leq q \leq \kappa_{1}$. Also, since $\left\langle u, K^{q} v\right\rangle=0$ for $u \in \mathfrak{L}^{-}$and $v \in V_{1}$, we see that $K^{q} V_{1} \subset \mathfrak{L}^{+}$, and hence it follows that $K^{2 q}$ is a positive operator in $V_{1}[12$, p. 147].

Definition 4.1. Let $q$ be the integer defined in the preceding paragraph. Then we say that the pair $(L, \omega)$ satisfies the condition $\left(C_{0}\right)$ if: $(1) \mid \Omega^{+} \backslash$ int $\Omega^{+} \mid=$ $\mid \Omega^{-} \backslash$ int $\Omega^{-} \mid=0$ and (2) $\omega$ and the $a_{\alpha \beta}$ (see 1.1)) are sufficiently smooth on $\Omega^{\#}=$ int $\Omega^{+} \cup$ int $\Omega^{-}$so that for any $\varphi \in C_{0}^{\infty}\left(\Omega^{\#}\right)$ we have $L \varphi \in \mathfrak{H}$ and if we define the functions $\left\{\varphi_{j}\right\}_{1}^{4 q}$ recursively by putting $\varphi_{j}(x)=(\omega(x))^{-1}\left(L \varphi_{j-1}\right)(x)$ for $x \in \Omega^{\#}, \varphi_{j}(x)=0$ for $x \in \Omega \backslash \Omega^{\#}$, where $\varphi_{0}=\varphi$, then we also have $L \varphi_{j} \in \mathfrak{H}$ for $j=1, \ldots,(4 q-1)$ and $\varphi_{j} \in H^{m}(\Omega)$ for $j=1, \ldots, 4 q$.

We note that in order for condition (2) in the above definition to be satisfied it is sufficient that $a_{\alpha \beta} \in C^{q^{\#}+|\alpha|, 1}\left(\Omega^{\#}\right)$ and that $\omega \in C^{q^{\#}, 1}\left(\Omega^{\#}\right)$, where $q^{\#}=$ $(8 q-1) m-1$. Observe also that for $j=0, \ldots, 4 q, \varphi_{j}$ has compact support which is contained in $\Omega^{\#}$ and $\varphi_{j} \in V$, while $\varphi_{j} \in D(A)$ for $j=0, \ldots,(4 q-1)$ and $A \varphi_{j}=T \varphi_{j+1}$.

Theorem 4.9. Suppose that $\operatorname{ker} T \cap V$ is degenerate with respect to the inner product $B($,$) . Suppose also that the pair (L, \omega)$ satisfies the condition $\left(\mathrm{C}_{0}\right)$. Then the problem (1.3-4) has infinitely many positive (resp. negative) eigenvalues. Moreover, the eigenvectors of (1.3-4) together with their associated vectors, if any, are complete in $L^{2}\left(\Omega^{+} \cup \Omega^{-}\right)$and if $\omega(x)$ satisfies the condition (C) of $\S 3$, then they are also complete in $L^{2}\left(\Omega^{+} \cup \Omega^{-} ;|\omega(x)| d x\right)$.

Proof. Let us fix our attention upon a particular fundamental decomposition of the Pontrjagin space $V_{1}$ and let $J$ denote the fundamental symmetry corresponding to this decomposition [12, p. 52]. Then when $V_{1}$, considered only as a vector space, is equipped with the inner product $\langle,\rangle_{J}=\langle J \cdot, \cdot\rangle$, it becomes a Hilbert space and the Hilbert topology of $V_{1}$ induced by the norm $\langle x, x\rangle_{J}^{1 / 2}$ is precisely the strong topology of $V_{1}$. If we now consider $V_{1}$ as such a Hilbert 
space and $K$ as an operator acting in $V_{1}$, then it follows that $K^{2 q}$ is a compact operator which is strongly symmetrisable with respect to the bounded selfadjoint operator $J\left[48\right.$, p. 371] and $S=J K^{2 q} \geq 0$. Since we know from above that the eigenvalues of $K$ must necessarily be real, we conclude from Lemma 4.1 and [48, Theorem 5, p. 420] that $K^{2 q}$ has infinitely many nonzero eigenvalues which are necessarily positive. Hence $K$ has infinitely many nonzero eigenvalues. We denote these nonzero eigenvalues by $\left\{\lambda_{j}\right\}_{1}^{\infty}$, where each $\lambda_{j}$ is repeated according to its multiplicity, and denote by $\left\{u_{j}\right\}_{1}^{\infty}$ the corresponding sequence of eigenvectors satisfying $\left(u_{j}, u_{k}\right)_{T}=\delta_{j k} \operatorname{sgn} \lambda_{j}$, where $\delta_{j k}$ is the Kronecker delta (see Remark 4.1 and the statements following Theorem 4.7).

Next let $\varphi \in C_{0}^{\infty}\left(\Omega^{\#}\right)$ (see Definition 4.1). Then it follows from the foregoing results that $\varphi=v_{0}+w_{0}$, where $v_{0} \in M_{0}(\dot{+}) M$ and $w_{0} \in V_{1}$. Moreover, if $M_{0}(\dot{+}) M \neq 0$, then it follows from $\S 2$, Theorems 4.4-5, and Remark 4.1 that there exist the vectors $\left\{v_{j}\right\}_{1}^{4 q}$ contained in $M_{0}(\dot{+}) M$ such that $T v_{j}=A v_{j-1}$. Putting $v_{j}=0$ for $j=1, \ldots, 4 q$ if $M_{0}(\dot{+}) M=0$ and defining $\left\{\varphi_{j}\right\}_{1}^{4 q}$ as in Definition 4.1, let $w_{j}=\varphi_{j}-v_{j}$ for $j=1, \ldots, 4 q$. Then $w_{j} \in V_{1}$ for $j=0, \ldots, 4 q, A w_{j}=T w_{j+1}$ for $j=0, \ldots,(4 q-1)$, and hence $w_{0}=K^{4 q} w_{4 q}=\sum_{j=1}^{\infty}\left\langle w_{0}, u_{j}\right\rangle u_{j}$ [48, Theorem 10, p. 422]. Since $C_{0}^{\infty}\left(\Omega^{\#}\right)$ is dense in $\mathfrak{H}^{+}=L^{2}\left(\Omega^{+} \cup \Omega^{-}\right)$, we conclude from the remarks following Theorem 4.7 that the eigenvectors and associated vectors of the problem (1.3-4) are complete in $\mathfrak{H}^{\dagger}$. If $\omega$ satisfies the condition $(\mathrm{C})$, then $C_{0}^{\infty}\left(\Omega^{\#}\right)$ is dense in $\mathfrak{H}^{\#}=$ $L^{2}\left(\Omega^{+} \cup \Omega^{-} ;|\omega(x)| d x\right)$, and hence, since the mapping $T: V_{1} \rightarrow \mathfrak{H}^{\#}$ is continuous, we conclude that the eigenvectors and associated vectors of (1.3-4) are complete in $\mathfrak{H}^{\#}$.

It remains only to prove that $K$ has infinitely many positive (resp. negative) eigenvalues in $V_{1}$. To this end let us observe that for $u \in V_{1}^{\#}=R\left(K^{4 q}\right)$ we have $u=\sum_{j=1}^{\infty} \lambda_{j}^{-1}\left(u, u_{j}\right)_{T} u_{j}$ and $(u, u)_{T}=\sum_{j=1}^{\infty}\left|\lambda_{j}\right|^{-2} \operatorname{sgn} \lambda_{j}\left|\left(u, u_{j}\right)_{T}\right|^{2}$. Hence if we now consider $V_{1}^{\#}$ only as a vector space and equip it with the inner product $(,)_{T}$, then it follows that $V_{1}^{\#}$ is positive definite if all the $\lambda_{j}$ are positive and the orthogonal direct sum of a finite dimensional negative definite subspace and a positive definite subspace if all but a finite number of the $\lambda_{j}$ are positive. Hence if we suppose that at most a finite number of the $\lambda_{j}$ are negative and consider $V^{\#}=M_{0}(\dot{+}) M(\dot{+}) V_{1}^{\#}$ only as a vector space and equip it with the inner product $(,)_{T}$, then it follows from the foregoing results and [12, Corollary 11.8, p. 26] that $V^{\#}$ is either positive definite or the orthogonal direct sum of a finite dimensional negative definite subspace and a positive definite subspace. On the other hand, since it follows from above that $V^{\#}$ contains negative definite subspaces of arbitrary large dimension, we may argue as in the proof of [12, Theorem 10.1, p. 50] to arrive at a contradiction. Hence infinitely many of the $\lambda_{j}$ are negative, and similarly we can show that infinitely many of the $\lambda_{j}$ are positive to complete the proof of the theorem. 
Acknowledgment. The author wishes to thank the referee for his valuable suggestions and for making him aware of some of the literature cited in the list of references.

\section{REFERENCES}

1. R. A. Adams, Sobolev spaces, Academic, New York, 1975.

2. S. Agmon, The coerciveness problem for integro-differential forms, J. Analyse Math. 6 (1958), 183-223.

3. __ Remarks on self-adjoint and semi-bounded elliptic boundary value problems, Proc. Internat. Sympos. on Linear Spaces (Jerusalem, 1960), Pergamon, Oxford, 1961, pp. 1-13.

4. __ On the eigenfunctions and on the eigenvalues of general elliptic boundary value problems, Comm. Pure Appl. Math. 15 (1962), 119-147.

5. ___ Lectures on elliptic boundary value problems, Van Nostrand, Princeton, N. J., 1965.

6. R. Beals, Indefinite Sturm-Liouville problems and half-range completeness, J. Differential Equations 56 (1985), 391-407.

7. P. A. Binding and K. Seddighi, On root vectors of self-adjoint pencils, J. Funct. Anal. 70 (1987), 117-125.

8. M. S. Birman and M. Z. Solomjak, Spectral asymptotics of nonsmooth elliptic operators. II, Trans. Moscow Math. Soc. 28 (1973), 1-32.

9. __ Asymptotic behaviour of the spectrum of differential equations, J. Soviet Math. 12 (1979), 247-282.

10. Asymptotics of the spectrum of variational problems on solutions of elliptic equations, Siberian Math. J. 20 (1979), 1-15.

11. _ Quantitative analysis in Sobolev imbedding theorems and applications to spectral theory, Amer. Math. Soc. Transl. (2) 114 (1980).

12. J. Bognár, Indefinite inner product spaces, Springer-Verlag, New York, 1974.

13. D. G. De Figueiredo, Positive solutions of semilinear elliptic problems, Lecture Notes in Math., vol. 957, Springer-Verlag, New York, 1982, pp. 34-87.

14. M. Faierman, The eigenvalues of a multiparameter system of differential equations, Appl. Anal. 19 (1985), 275-290.

15. __ Expansions in eigenfunctions of a two-parameter system of differential equations. II, Quaestiones Math. 10 (1987), 217-249.

16. M. Faierman and G. F. Roach, Full and half-range eigenfunction expansions for an elliptic boundary value problem involving an indefinite weight, Proc. 1987 Equadiff Conference, Lecture Notes in Pure and Appl. Math., vol. 118, Dekker, New York and Basel, 1989, pp. 231-236.

17. J. Fleckinger and A. B. Mingarelli, On the eigenvalues of non-definite elliptic operators, Math. Studies, vol. 92, North-Holland, Amsterdam, 1984, pp. 219-227.

18. J. Fleckinger-Pelle, Asymptotics of eigenvalues for some non-definite elliptic problems, Lecture Notes in Math., vol. 1151, Springer-Verlag, Berlin and New York, 1985, pp. 148-156.

19. J. Fleckinger and M. L. Lapidus, Eigenvalues of elliptic boundary value problems with an indefinite weight function, Trans. Amer. Math. Soc. 295 (1986), 305-324.

20. __ Remainder estimates for the asymptotics of elliptic eigenvalue problems with indefinite weights, Arch. Rational Mech. Anal. 98 (1987), 329-356.

21. H. E. Gerlach, Beitrag zur Theorie einer Klasse von Randwertaufgaben, Thesis, Saarbrücken, 1962.

22. D. Gilbarg and N. S. Trudinger, Elliptic partial differential equations of second order, 2 nd ed., Springer-Verlag, Berlin and New York, 1983. 
23. O. Haupt, Untersuchungen über Oszillationstheoreme, Teubner, Leipzig, 1911.

24. __ Über eine Methode zum Beweise von Oszillationstheoreme, Math. Ann. 76 (1915), 67-104.

25. P. Hess, On the relative completeness of the generalized eigenvectors of elliptic eigenvalue problems with indefinite weight functions, Math. Ann. 270 (1985), 467-475.

26. H. Hilb, Eine Erweiterung des Kleinschen Oszillationstheorems, Jabresbericht d. d. Math. Ver. 16 (1907), 279-285.

27. D. Hilbert Grundzüge einer allgemeinen Theorie der linearen Integralgleichungen, Chelsea, New York, 1953.

28. E. Hölmgren, Über Randwertaufgaben bei einer linearen Differentialgleichungen zweiter Ordnung, Ark. Mat. Astronom. Fysik 1 (1904), 401-417.

29. L. Hörmander, Linear partial differential operators, Springer-Verlag, Berlin and New York, 1976.

30. __ Uniqueness theorems for second order elliptic differential equations, Comm. Partial Differential Equations 8 (1983), 21-64.

31. I. S. Iohvidov, On the spectra of Hermitian and unitary operators in a space with an indefinite metric, Dokl. Akad. Nauk SSSR 71 (1950), 225-228.

32. K. Jörgens, Spectral theory of second order ordinary differential equations, Aarhus Universitet Lecture Notes, 1964.

33. T. Kato, Perturbation theory for linear operators, 2nd ed., Springer-Verlag, Berlin and New York, 1976.

34. J. L. Lions, Problèmes aux limites dans les équations aux derivées partielles, Les Presses de l'Université de Montréal, Montréal, 1965.

35. J. L. Lions and E. Magenes, Non-homogeneous boundary value problems and applications, vol. I, Springer-Verlag, Berlin and New York, 1972.

36. A. I. Mal' cev, Foundations of linear algebra, Freeman, San Francisco, Calif., 1963.

37. A. B. Mingarelli, Indefinite Sturm-Liouville problems, Lecture Notes in Math., vol. 964, Springer-Verlag, Berlin and New York, 1983, pp. 519-528.

38. __ On the existence of nonsimple real eigenvalues for general Sturm-Liouville problems, Proc. Amer. Math. Soc. 89 (1983), 457-460.

39. A survey of the regular weighted Sturm-Liouville problem-the non-definite case, Proc. Workshop on Applied Differential Equations, Tsinghua University, Beijing, 3-7 June 1985 (X. Shutie and P. Fuquan eds.), World Scientific Publishing, Singapore and Philadelphia, Pa., 1986, pp. 109-137.

40. Ȧ. Pleijel, Sur la distribution des valeurs propres de problèmes régis par l'équation $\Delta u+$ $\lambda k(x, y) u=0$, Ark. Mat. Astronom. Fysik 29B (1942), 1-8.

41. R. G. D. Richardson, Theorems of oscillation for two linear differential equations of the second order with two parameters, Trans. Amer. Math. Soc. 13 (1912), 22-34.

42. __ Über die notwendigen und hindreichenden Bedingungen für das Bestehen eines Kleinschen Oszillationstheorems, Math. Ann. 73 (1913), 289-304 (errata, Math. Ann. 74 (1913) 312 ).

43. __ Contributions to the study of oscillation properties of the solutions of linear differential equations of the second order, Amer. J. Math. 40 (1918), 283-316.

44. G. V. Rozenbljum, The distribution of the discrete spectrum for singular differential operators, Soviet Math. Dokl. 13 (1972), 245-249.

45. G. Stampacchia, Equations elliptiques du second ordre à coefficients discontinus, Les Presses de l'Université de Montréal, Montréal, 1966. 
46. H. F. Weinberger, Variational methods for eigenvalue approximation, CBMS Regional Conf. Ser. Appl. Math., vol. 15, SIAM, Philadelphia, Pa., 1974.

47. K. Yosida, Functional analysis, 5th ed., Springer-Verlag, New York, 1978.

48. A. C. Zaanen, Linear analysis, North-Holland, Amsterdam, 1953.

Department of Mathematics, University of Witwatersrand, Johannesburg, South AFRICA 